\title{
On List Update with Locality of Reference*
}

\author{
Susanne Albers ${ }^{\dagger} \quad$ Sonja Lauer ${ }^{\ddagger}$
}

\begin{abstract}
We present a comprehensive study of the list update problem with locality of reference. More specifically, we present a combined theoretical and experimental study in which the theoretically proven and experimentally observed performance guarantees of algorithms match or nearly match.

In the first part of the paper we introduce a new model of locality of reference that closely captures the concept of runs, representing sequences of requests to the same item. Using this model we develop refined theoretical analyses of popular list update algorithms. The second part of the paper is devoted to an extensive experimental study in which we have tested the algorithms on traces from benchmark libraries. It shows that the theoretical and experimental bounds differ by just a few percent.

Our new theoretical bounds are substantially lower than those provided by standard competitive analysis. Another result is that the well-known Move-To-Front strategy exhibits the best performance. Its refined competitive ratio tends to 1 as the degree of locality in a request sequence increases. This confirms that Move-To-Front is the method of choice in practice.
\end{abstract}

\section{Introduction}

The list update problem is one of the most extensively studied online problems, with a tremendous body of literature published over the past 40 years. The problem has been investigated with respect to both averagecase and worst-case competitive analysis. We refer the reader to $[1,4,5,6,12,18,27,30,32,35,36,38,41]$ for a selection of some key results.

The list update problem consists in maintaining a set of items as an unsorted linear list. More specifically, a linear linked list of items is given. A list update algorithm is presented with a sequence of requests that must be served in their order of occurrence. Each request specifies an item in the list. In order to serve a request, a list update algorithm must access the requested item, i.e. it has to start at the front of the list and search linearly through the items until the desired item is found. Accessing the $i$-th item in the list incurs a cost of $i$. Immediately after an access, the requested item may be moved at no extra cost to any position closer to the front of the list. These exchanges are called free exchanges. All other exchanges of two consecutive items in the list cost 1 and are called paid exchanges. The goal is to serve the request sequence so that the total cost is as small as possible. We emphasize that this is the standard cost model, see also [38]. Of particular interest are online algorithms that serve each request without knowledge of any future requests.

While early work on the list update problem evaluated online algorithms assuming that requests are generated according to probability distributions, research over the past 20 years has focused on competitive analysis [38]. Here an online algorithm is compared to an optimal offline algorithm. Given a request sequence $\sigma$,

\footnotetext{
*Work supported by the Deutsche Forschungsgemeinschaft and the European Research Council (ERC), Grant Agreement No. 691672.

${ }^{\dagger}$ Department of Computer Science, Technische Universität München, Boltzmannstr. 3, 85748 Garching, Germany, albersin.tum.de.

${ }^{\ddagger}$ Department of Computer Science, University of Freiburg, Georges Köhler Allee 79, 79110 Freiburg, Germany. sonja.lauer@informatik.uni-freiburg.de
} 
let $A(\sigma)$ denote the cost incurred by online algorithm $A$ in serving $\sigma$, and let $O P T(\sigma)$ denote the optimum offline cost. Algorithm $A$ is called $c$-competitive if there exists a constant $\alpha$ such that $A(\sigma) \leq c \cdot O P T(\sigma)+\alpha$ holds for all $\sigma$ and all size lists.

In 1985 Sleator and Tarjan proved that the Move-To-Front algorithm is 2-competitive [38]. This elegant strategy simply moves an item to the front of the list whenever it is requested. Since then, algorithms with an improved competitiveness have been developed. While the competitive ratios are of course constant, there is a substantial gap between the theoretical bounds and the performance ratios of the algorithms observed in practice. Moreover, Move-To-Front often outperforms other strategies, see e.g. [10, 12]. The reason is that competitive analysis considers arbitrary request sequences, whereas sequences arising in practice have a special structure: They exhibit locality of reference, meaning that at any point in time only a small set of items is referenced.

There has been considerable research interest in studying the paging problem with locality of reference [3, $7,14,20,25,26,31,33$ ] because, in paging, the gap between the theoretical and experimental performance values is even super-constant. However, hardly any work has been presented for the classical list update problem. In fact, references [10,29] point out that locality is an essential aspect in the list update problem and that a good model is required to properly evaluate the performance of algorithms.

Previous results: We focus on the results that have been developed in the framework of competitive analysis. As mentioned above Sleator and Tarjan [38] showed that Move-To-Front is 2-competitive. This is the best factor deterministic online algorithms can achieve [32]. Bachrach and El-Yaniv [9] devised deterministic $M R I$ and $P R I$ families of algorithms. These families attain competitive ratios of 2 and 3 , respectively. We next turn to randomized algorithms. The first randomized strategy was presented by Irani [30]. Her Split algorithm is 1.9375-competitive. Reingold et al. [35] presented an elegant BIT algorithm that is 1.75-competitive. This factor is substantially below the deterministic bound of 2 . The BIT algorithm can be generalized to a family of Counter strategies [35]. A Timestamp family of algorithms was developed in [1]. It achieves a competitiveness equal to the Golden Ratio $\Phi \approx 1.62$. The best randomized algorithm currently known is $C O M B$ which is 1.6competitive [4]. Interestingly, COMB is a combination of BIT and a (deterministic) element of the Timestamp family. The factor of 1.6 is close to best lower bound of 1.50084 developed by Ambühl et al. [6] on the performance of randomized list update algorithms.

Experimental studies for the list update problem have been presented by Rivest [36], Bentley and McGeoch [12] and Bachrach et al. [10]. They analyzed popular algorithms on request sequences generated by probability distributions and Markov sources, on sequences derived from text and Pascal files as well as on sequences extracted from the Calgary Corpus [17]. The results are not unanimous. A conclusion is that the ranking of algorithms depends on the degree of locality in the input.

The only prior work addressing list update with locality of reference was a paper by Angelopoulos et al. [8]. They adapted a locality model [3] introduced for the paging problem and proved that Move-To-Front is superior to other algorithms.

Our contribution: We present a comprehensive study of the list update problem with locality of reference. The goal is to provide a refined analysis of the problem in which theoretical and empirical results match or nearly match. To this end our study integrates theoretical and experimental work.

First, in Section 2, we introduce a new model of locality of reference that is based on the natural concept of runs. A run is a sequence of requests to the same item. We define a number of parameters that characterize request sequences in terms of the occurrence of long runs. Using these parameters we will be able to accurately estimate the performance of list update algorithms. We also define a model of so-called $\lambda$-locality that characterizes classes of input sequences with respect to their degree of locality. Loosely speaking, the more long runs there are, the higher the locality. As we shall see, our new concepts properly capture locality of reference in the list update problem, both from a theoretical and practical point of view.

In Section 3 we present refined theoretical analyses of list update algorithms. We concentrate on the most 
popular strategies that have received the most attention recently, namely Move-To-Front, BIT and COMB. In order to be able to analyze $C O M B$, we have also evaluated a member of the Timestamp family. Of course, we have also investigated an optimal offline strategy. For each algorithm we have analyzed the total service cost incurred on a request sequence, where cost is expressed in terms of our new locality parameters. Interestingly, for Move-To-Front our cost analysis is exact, i.e. our locality model is powerful enough to exactly quantify Move-To-Front's service cost on any request sequence. Furthermore, for each online algorithm, we have evaluated its performance relative to that of an optimal offline algorithm. Here Move-To-Front achieves an excellent performance ratio and responds well to locality of reference: The competitiveness even tends down to 1 as the degree of locality increases. This does not hold true for the other online algorithms.

In Section 4, we present a comprehensive experimental study in which we have evaluated our list update algorithms on real-world traces from benchmark libraries. Obviously, the list update problem is a solution to the classic dictionary problem. In this context, in practice, requests are memory accesses. Secondly, list update has interesting applications in data compression, see e.g. $[13,16]$. For instance, the open source data compression program bzip2 relies on Move-To-Front encoding in combination with a preceding Burrows Wheeler transformation. Therefore, in our experiments we consider as input (a) memory access strings (47 traces) and (b) sequences arising in data compression routines (44 traces). In our tests we first analyze the traces with respect to their locality characteristics. It shows that the parameters introduced in Section 2 are indeed sensible.

Next, in the experiments, for each algorithm and each input sequence, we have computed the total service cost. Furthermore, for each online algorithm and each input, we have determined the experimentally observed competitiveness, which is the total service cost of the algorithm divided by the total cost incurred by an optimal offline strategy. Since the offline version of the list update problem is NP-hard, we have approximated the optimum service cost by that of the pairwise optimum, see Section 4 for details. In general, our theoretically proven and experimentally observed bounds are very close and differ by just a few percent. As for the total service cost, Move-To-Front exhibits an error of 0 because our theoretical bound is exact. For the other three online algorithms BIT, Timestamp and $C O M B$, the average relative error between the theoretical and experimentally observed values is $3-4 \%$ on the memory access traces and $7-9 \%$ on the data compression sequences. As far as performance ratios relative to the optimum are concerned, the average relative errors between our theoretical bounds and the experimentally observed competitive ratios are a bit higher. MoveTo-Front exhibits average relative errors of $0.3 \%$ on the memory traces and of $0.7 \%$ on the data compression sequences. The other three strategies incur average errors of $3-4 \%$ on the memory traces and of $8-10 \%$ on the data compression sequences.

In our study, the theoretical and experimental performance ratios of the algorithms are much lower than the corresponding standard competitive ratios. In particular, Move-To-Front shows the best performance with ratios in the range of 1.2-1.3. This confirms that Move-To-Front is the method of choice in practice.

We finally remark that our study does not address the algorithms Transpose and Frequency Count as they to not achieve constant competitive ratios [38].

Subsequent work: After the conference publication of our paper, list update with locality of reference has been studied in [19, 22, 21]. Dorrigiv et al. [19] propose a locality model based on Denning's working sets and analyze the performance of algorithms using a certain non-locality parameter. They show that Move-ToFront is an optimal strategy with respect to this parameter. Moreover, the analysis separates Move-To-Front from other strategies. However, no experimental study is conducted, comparing the theoretical bounds to those observed in practice. Dorrigiv and López-Ortiz [22, 21] study list update algorithms under probability distributions that exhibit locality of reference. They analyze the expected service cost of various algorithms and show that Move-To-Front is the best strategy in this framework. A study of list update algorithms in data compression was presented by Dorrigiv et al. [23, 24]. 


\section{A new model for locality of reference}

Informally speaking, a request sequence exhibits locality of reference if, at any time, it references only a small set of items. If an item is requested, it is likely to be requested again soon. This description suggests the concept of runs, where a run is a subsequence of requests to the same item. In the best case, when there is a high degree of locality, an item is requested many times in a row before a different element is referenced. Unfortunately, real-world request sequences may contain only few of these pure long runs. However, long runs may occur if we focus on small item sets and, in particular, on item pairs: If, at any time, item $x$ is more relevant than $y$, then this relation is likely to hold also in the near future and we encounter several requests to $x$ before the next reference to $y$ arises. Thus long runs occur if we project request sequences to smaller item sets or item pairs. A request sequence exhibits a high degree of locality if a substantial portion of the requests belongs to long runs. In the following we introduce a formal model of locality of reference based on this generalized notion of runs.

Let $L$ be the set of items in the list to be maintained. Consider a fixed request sequence $\sigma$. For any two items $x, y \in L$ with $x \neq y$, let $\sigma_{x y}$ be the request sequence that is derived from $\sigma$ when deleting all requests that are neither to $x$ nor to $y$, i.e. only the requests to $x$ and $y$ survive. Any maximal subsequence of consecutive requests to the same item in $\sigma_{x y}$ is called a run. Let $r\left(\sigma_{x y}\right)$ be the number of runs in $\sigma_{x y}$. A run is short if it consists of one request only. A run consisting of at least two requests is long. Let $s\left(\sigma_{x y}\right)$ and $l\left(\sigma_{x y}\right)$ denote the number of short and long runs, respectively, in $\sigma_{x y}$. Then $s\left(\sigma_{x y}\right)+l\left(\sigma_{x y}\right)=r\left(\sigma_{x y}\right)$.

On long runs online algorithms typically perform well, relative to an optimal offline algorithm. In order to properly evaluate our algorithms, we need some further definitions. A long run $\rho$ is called a prefixed long run if it is preceded by one or more short runs; otherwise $\rho$ is called an independent long run. Again let $l_{p}\left(\sigma_{x y}\right)$ and $l_{i}\left(\sigma_{x y}\right)$ be the number of prefixed and independent long runs, respectively. We have $l_{p}\left(\sigma_{x y}\right)+l_{i}\left(\sigma_{x y}\right)=$ $l\left(\sigma_{x y}\right)$.

Consider two long runs $\rho^{\prime}$ and $\rho$ such that $\rho^{\prime}$ occurs earlier than $\rho$ in $\sigma_{x y}$. Run $\rho^{\prime}$ immediately precedes $\rho$ if the last request of $\rho^{\prime}$ is followed by the first request of $\rho$ in $\sigma_{x y}$ or if $\rho^{\prime}$ and $\rho$ are separated by short runs only. A long run $\rho$ which is not equal to the first long run in $\sigma_{x y}$ represents a long run change if $\rho$ and the immediately preceding long run $\rho^{\prime}$ reference different items. The first long run $\rho$ in $\sigma_{x y}$ represents a long run change if $\rho$ and the first request of $\sigma_{x y}$ reference the same item (imagining that $\sigma_{x y}$ was preceded by a long run to just the other item of $\{x, y\})$. Let $l_{c}\left(\sigma_{x y}\right)$ be the number of long run changes in $\sigma_{x y}$. We have $l_{c}\left(\sigma_{x y}\right) \leq l\left(\sigma_{x y}\right)$. Furthermore, $l_{i}\left(\sigma_{x y}\right) \leq l_{c}\left(\sigma_{x y}\right)$ because each independent long run, except for possibly the first one, is immediately preceded by another long run, which references a different item. The number of long run changes will be particularly important in lower bounding the cost incurred by an optimal offline algorithm. Hence, it will allow us to derive good upper bounds on the relative performance ratios of online strategies. Table 1 summarizes the various parameters for a projected sequence $\sigma_{x y}$.

As an example, consider $\sigma_{x y}=$ xxyyyxyxyxxxxyxxx. The sequence consists of nine runs, five of them are short and the remaining four are long. That is $r\left(\sigma_{x y}\right)=9, s\left(\sigma_{x y}\right)=5$ and $l\left(\sigma_{x y}\right)=4$. The runs $x x x x$ and $x x x$ are prefixed long runs so that $l_{p}\left(\sigma_{x y}\right)=2$ and $l_{i}\left(\sigma_{x y}\right)=2$. All long runs, except for the last one, represent a long run change, i.e. $l_{c}\left(\sigma_{x y}\right)=3$. On the other hand, if $\sigma_{x y}$ started with a short run to $x$, then the following long run yyy would not be a long run change.

So far we have defined a number of values for a particular request sequence $\sigma_{x y}$. We now sum these values over all pairs of items $x$ and $y$. For any pair $x, y \in L$ with $x \neq y$ and for any value $v \in\left\{r, s, l, l_{i}, l_{p}, l_{c}\right\}$, let $v(\sigma)=\sum_{\{x, y\} \subseteq L, x \neq y} v\left(\sigma_{x y}\right)$. For instance, $r(\sigma)$ is the total number of runs in $\sigma$, while $s(\sigma)$ and $l(\sigma)$ represent the total number of short and long runs, respectively, in $\sigma$. In the experiments (Section 4 ) it shows that all of the parameters are sensible. Typically, $60 \%$ of the runs are long runs. Most of them are independent long runs. Moreover, the value $l_{c}$ is quite expressive. The ratio $l_{c} / l$ is usually $5 \%$ to $10 \%$ higher than $l_{i} / l$.

All the definitions presented so far refer to a given request sequence $\sigma$ and, using these definitions, we will be able to accurately evaluate the performance of list update algorithms on such a $\sigma$. Next, we introduce 


\begin{tabular}{|l|l|}
\hline Parameter & Count \\
\hline$r\left(\sigma_{x y}\right)$ & runs \\
$s\left(\sigma_{x y}\right)$ & short runs \\
$l\left(\sigma_{x y}\right)$ & long runs \\
$l_{p}\left(\sigma_{x y}\right)$ & prefixed long runs \\
$l_{i}\left(\sigma_{x y}\right)$ & independent long runs \\
$l_{c}\left(\sigma_{x y}\right)$ & long run changes \\
\hline
\end{tabular}

Table 1: The parameters of the locality model, for any $\sigma_{x y}$. For a request sequence $\sigma$, the parameters are summed over all pairs $x \neq y$.

a model of locality of reference that applies to classes of request sequences which may be generated by a particular application. Intuitively, request sequences exhibit a high degree of locality if there are many long runs. However, in order to obtain meaningful results we have to work with a refined definition. Again, the number of long run changes is crucial. We say that a class $\Sigma$ of request sequences exhibits $\lambda$-locality, for some $0 \leq \lambda \leq 1$, if for any $\sigma \in \Sigma$ inequality $l_{c}(\sigma) / r(\sigma) \geq \lambda$ holds, i.e. the number of long run changes represents at least a fraction of $\lambda$ among all the runs. Note that, for a given request sequence, $l_{c}(\sigma)$ accounts for all the independent long runs and, depending on the input, for a smaller or larger fraction of the prefixed long runs. If a request sequence consists of long runs only, we have $\lambda=1$.

An alternative, perhaps more intuitive definition would be to set $\lambda$ as $l(\sigma) / r(\sigma)$. However with this definition we would not be able to derive good bounds because the analysis of an optimal offline algorithm $O P T$ crucially depends on $l_{c}(\sigma)$. A reader may wonder why our locality model does not incorporate the length of long runs. This parameter is irrelevant for algorithms performance because after the second request of a long run competitive algorithms have moved the referenced item ahead of the other item in the list and no further cost is incurred on the run. We finally remark that our new locality model, based on runs, is different from models introduced for paging, see again $[3,7,14,20,33]$. The latter usually model working sets and, in particular, working set sizes over certain time intervals.

\section{Analyzing online and offline algorithms}

In this section we present refined theoretical analyses of list update algorithms. We first revisit a general analysis framework based on item pairs. Then we lower bound the cost of an optimal offline algorithm. This estimate will be crucial to evaluate the performance of online algorithms.

\subsection{Basic cost analysis}

We show that the cost incurred by any online or offline list update algorithm $A$ on a request sequence $\sigma$ can be evaluated by considering pairs of items. This reduction is not new, but the reduction shown here incorporates for the first time paid exchanges that an algorithm may perform.

Let $m=|\sigma|$ be the length of $\sigma$ and $\sigma(t)$ be the request posed at time $t, 1 \leq t \leq m$. The cost incurred by $A$ in accessing $\sigma(t)$ is 1 plus the number of items that precede item $\sigma(t)$ in the list at time $t$. Additionally, $A$ may perform paid exchanges. For any item $x \in L$ and any time $t$, define $A_{x}(t, \sigma)=1$ if $x$ precedes item $\sigma(t)$ in the list at time $t$; otherwise $A_{x}(t, \sigma)=0$. We note that $A_{x}(t, \sigma)=0$ for any $t$ with $\sigma(t)=x$. Furthermore, let $A_{p}(t, \sigma)$ denote the number of paid exchanges performed by $A$ at time $t$. Using these definitions, the cost 
incurred by any online or offline list update algorithm $A$ can be expressed as

$$
\begin{aligned}
A(\sigma) & =\sum_{t=1}^{m}\left(\sum_{x \in L} A_{x}(t, \sigma)+A_{p}(t, \sigma)\right)+m \\
& =\sum_{x \in L} \sum_{t=1}^{m} A_{x}(t, \sigma)+\sum_{t=1}^{m} A_{p}(t, \sigma)+m \\
& =\sum_{x \in L} \sum_{y \in L} \sum_{t: \sigma(t)=y} A_{x}(t, \sigma)+\sum_{t=1}^{m} A_{p}(t, \sigma)+m \\
& =\sum_{\substack{\{x, y\} \subseteq L \\
x \neq y}} \sum_{\substack{t: \\
\sigma(t) \in\{x, y\}}}\left(A_{x}(t, \sigma)+A_{y}(t, \sigma)\right)+\sum_{t=1}^{m} A_{p}(t, \sigma)+m .
\end{aligned}
$$

Let $A_{p, x y}(\sigma)$ be the total number of paid exchanges performed by $A$ to change the relative order of $x$ and $y$ in the list while serving $\sigma$. Then

$$
A(\sigma)=\sum_{\substack{\{x, y\} \subseteq L \\ x \neq y}}\left(\sum_{\substack{t: \\ \sigma(t) \in\{x, y\}}}\left(A_{x}(t, \sigma)+A_{y}(t, \sigma)\right)+A_{p, x y}(\sigma)\right)+m .
$$

Furthermore, let $A_{x y}(\sigma)=\sum_{t: \sigma(t) \in\{x, y\}}\left(A_{x}(t, \sigma)+A_{y}(t, \sigma)\right)+A_{p, x y}(\sigma)$. This term intuitively represents the cost incurred by items $x$ and $y$ on requests that are to either $x$ or $y$, plus the number of paid exchanges involving both $x$ and $y$. We remark that, for any $t$ with $\sigma(t) \in\{x, y\}$, the sum $A_{x}(t, \sigma)+A_{y}(t, \sigma)$ is either 0 or 1 depending on whether or not the requested item precedes the other item of the pair $\{x, y\}$. With the abbreviation $A_{x y}(\sigma)$ we obtain

$$
A(\sigma)=\sum_{\substack{\{x, y\} \subseteq L \\ x \neq y}} A_{x y}(\sigma)+|\sigma| .
$$

Suppose that algorithm $A$ serves $\sigma_{x y}$ on the two-item list that consists of $x$ and $y$ only and let $A\left(\sigma_{x y}\right)$ be the incurred cost in the partial cost model. In this model the cost of serving a request is equal to the number of items that precede the requested item in the current list, i.e. a request to the first item in the list costs 0 and a request to the second item in the list costs 1 . All online algorithms proposed in the literature for list update have the property that $A_{x y}(\sigma)=A\left(\sigma_{x y}\right)$, for any $x, y \in L$ with $x \neq y$. We will verify this property when studying online algorithms in the following sections. As for an optimal offline strategy $O P T$, inequality $O P T_{x y}(\sigma) \geq O P T\left(\sigma_{x y}\right)$ holds. Again we will verify this property in the sequel. Therefore, it will be convenient to study $A\left(\sigma_{x y}\right)$ instead of analyzing the (online or offline) cost $A_{x y}(\sigma)$.

For the analysis of $A\left(\sigma_{x y}\right)$ we will often partition $\sigma_{x y}$ into phases such that each phase ends with a long run; the last phase ends with the last request of $\sigma_{x y}$ if the last run happens to be short. Let $p_{x y}$ denote the number of phases in this partition and let $\pi(i)$ be the $i$ th phase, $1 \leq i \leq p_{x y}$. If $\pi(i)$ starts with a request to $x$, then the phase has one of the following two structures, depending on whether the last request of the phase is to $x$ or $y$.

$$
\begin{array}{ll}
\text { (a) }(x y)^{k} x^{l} \quad k \geq 0, l \geq 1 & \text { (b) }(x y)^{k} y^{l} \quad k \geq 1, l \geq 0
\end{array}
$$

If $\pi(i)$ starts with a request to $y$, the structures are symmetric. If the phase ends with a long run, we have $k \geq 0, l \geq 2$ in case (a) and $k \geq 1, l \geq 1$ in case (b).

Based on this phase partitioning, we introduce some definitions regarding the beginning and end of $\sigma_{x y}$ and $A$ 's list configuration. Let $f_{b}\left(\sigma_{x y}\right)$ be equal to 1 if the item first requested in $\sigma_{x y}$ precedes the other item of $\{x, y\}$ in the initial list; otherwise let $f_{b}\left(\sigma_{x y}\right)=0$. Moreover, let $f_{b}^{\prime}\left(\sigma_{x y}\right)$ be equal to 1 if $f_{b}\left(\sigma_{x y}\right)=1$ and $\sigma_{x y}$ starts with a short run followed by a long run; otherwise $f_{b}^{\prime}\left(\sigma_{x y}\right)=0$. Finally, let $f_{e}\left(\sigma_{x y}\right)$ be equal to 1 if 
the last phase consists of a single request and the referenced item is stored after the other item of $\{x, y\}$ in the current list when $A$ processes $\sigma_{x y}$ on the two-item list and reaches the beginning of the last phase; otherwise $f_{e}\left(\sigma_{x y}\right)=0$. Table 2 summarizes these additional parameters.

As an example, let $\sigma_{x y}=x y y y x x y$ and assume that $A$ is the Move-To-Front algorithm. Suppose that in the initial list $x$ is stored before $y$. In this case $f_{b}\left(\sigma_{x y}\right)=1$ and $f_{b}^{\prime}\left(\sigma_{x y}\right)=1$. Moreover, $f_{e}\left(\sigma_{x y}\right)=1$ because immediately before Move-To-Front serves the last run, $x$ is stored before $y$ in the current list.

\begin{tabular}{|l|l|}
\hline Indicator & Value \\
\hline$f_{b}\left(\sigma_{x y}\right)$ & 1 if item requested first is in front \\
$f_{b}^{\prime}\left(\sigma_{x y}\right)$ & 1 if additionally $\sigma_{x y}$ starts with short run followed by long run \\
$f_{e}\left(\sigma_{x y}\right)$ & 1 if $\sigma_{x y}$ ends with short run and item is in the back \\
\hline
\end{tabular}

Table 2: Summary of the additional parameters for $\sigma_{x y}$. Again they are to be summed over all pairs $x \neq y$.

Again we sum these definitions over item pairs. For any $x, y \in L$ with $x \neq y$ and for any value $v \in\left\{f_{b}, f_{b}^{\prime}, f_{e}\right\}$, let $v(\sigma)=\sum_{\{x, y\} \subseteq L, x \neq y} v\left(\sigma_{x y}\right)$. We remark that values $v(\sigma)$, with $v \in\left\{f_{b}, f_{b}^{\prime}, f_{e}\right\}$, will be needed to properly evaluate the cost of our investigated algorithms. However, when determining the performance of online algorithms relative to the optimal offline strategy, many of the terms will cancel. Furthermore, we observe that values $v(\sigma)$ with $v \in\left\{r, s, l, l_{c}, l_{p}, l_{i}\right\}$ may grow arbitrarily large as the length of $\sigma$ increases, whereas the other values $v(\sigma)$ with $v \in\left\{f_{b}, f_{b}^{\prime}, f_{e}\right\}$ are bounded by $|L|(|L|-1) / 2$.

\subsection{The cost of an optimal offline algorithm}

We lower bound the cost incurred by an optimal offline algorithm $O P T$ on a request sequence $\sigma$.

Lemma 1 The cost incurred by $O P T$ is at least $O P T(\sigma) \geq \frac{1}{2}\left(r(\sigma)+l_{c}(\sigma)+f_{e}(\sigma)\right)-f_{b}(\sigma)+|\sigma|$.

Proof. We first argue that $O P T_{x y}(\sigma) \geq O P T\left(\sigma_{x y}\right)$ holds for any fixed pair of items $x, y \in L$ with $x \neq y$, cf. also [30]. Suppose that we serve $\sigma_{x y}$ on the two-item list consisting of $x$ and $y$ by mimicking OPT's behavior when servicing $\sigma$ on the entire list of all items in $L$. More specifically, we change the relative order of $x$ and $y$ in the two-item list, using free or paid exchanges, whenever OPT does so in the entire list. This service schedule incurs a cost of $O P T_{x y}(\sigma)$ in the partial cost model, and the latter value cannot be smaller than the optimal cost $O P T\left(\sigma_{x y}\right)$ of serving $\sigma_{x y}$ on the two-item list. Hence we find $O P T_{x y}(\sigma) \geq O P T\left(\sigma_{x y}\right)$.

Using (1) we find $O P T(\sigma) \geq \sum_{\{x, y\} \subseteq L, x \neq y} O P T\left(\sigma_{x y}\right)+|\sigma|$. In the following we consider a fixed pair of items $x, y \in L$ with $x \neq y$ and will prove

$$
O P T\left(\sigma_{x y}\right) \geq \frac{1}{2}\left(r\left(\sigma_{x y}\right)+l_{c}\left(\sigma_{x y}\right)+f_{e}\left(\sigma_{x y}\right)\right)-f_{b}\left(\sigma_{x y}\right) .
$$

Summing (2) over all pairs of items and taking into account that $O P T(\sigma) \geq \sum_{\{x, y\} \subseteq L, x \neq y} O P T\left(\sigma_{x y}\right)+|\sigma|$, we derive the lemma. An optimal offline algorithm for serving $\sigma_{x y}$ on a two-item list consisting of $x$ and $y$ is easy to state: On the first request of each long run move the requested item to the front of the list. On any other request, do not change the position of the referenced item. No paid exchanges are used.

We will show that, essentially, $O P T\left(\sigma_{x y}\right) \geq \frac{1}{2}\left(r\left(\sigma_{x y}\right)+l_{c}\left(\sigma_{x y}\right)\right)$. However, in order to establish a correct and accurate lower bound, we have to consider the list configuration at the beginning and end of $\sigma_{x y}$, which is captured by $f_{b}\left(\sigma_{x y}\right)$ and $f_{e}\left(\sigma_{x y}\right)$, see again Table 2 .

In order to analyze $O P T\left(\sigma_{x y}\right)$ we partition $\sigma_{x y}$ into phases as described in Section 3.1. We analyze an arbitrary phase $\pi(i), 1 \leq i \leq p_{x y}$, and assume w.l.o.g. that the phase starts with a request to item $x$. Recall that $\pi(i)$ has one of the following two structures: (a) $(x y)^{k} x^{l}$ with $k \geq 0, l \geq 1$ or (b) $(x y)^{k} y^{l}$ with $k \geq 1, l \geq 0$. 
If $\sigma_{x y}$ consists of a single request to item, say $x$, then (2) is easy to see. We have $r\left(\sigma_{x y}\right)=1$ and $l_{c}\left(\sigma_{x y}\right)=0$. If $x$ is stored in front of $y$ in the initial list, then the cost incurred by OPT is 0 . Since $f_{b}\left(\sigma_{x y}\right)=1$ and $f_{e}\left(\sigma_{x y}\right)=0$, the right hand side of (2) is in fact smaller than 0 . On the other hand, if $x$ is stored after $y$ in the initial list, then the cost incurred by $O P T$ is 1 . We have $f_{b}\left(\sigma_{x y}\right)=0$ and $f_{e}\left(\sigma_{x y}\right)=1$ such that the right hand side of (2) is also equal to 1 . In the remainder of this analysis we assume that $\sigma_{x y}$ consists of more than one request.

For any $i$ with $1 \leq i \leq p_{x y}$, let $r(\pi(i))$ be the number of runs in $\pi(i)$. Furthermore, let $l_{c}(\pi(i))=1$ if phase $i$ ends with a long run and this long run represents a long run change. Otherwise we set $l_{c}(\pi(i))=0$. A long run change occurs in phase $i$ if and only if the phase has structure (a) with $l \geq 2$ : This holds because, if $i \geq 2$, phase $i-1$ ended with a long run of requests to item $y$ and only phase structure (a) ends with $x$. If $i=1$, then a long run ending phase 1 is the first long run in $\sigma_{x y}$ and represents a long run change if the referenced item is equal to the item first referenced in $\sigma_{x y}$. Finally, let $O P T(\pi(i))$ denote the cost incurred by $O P T$ when serving $\pi(i), 1 \leq i \leq p_{x y}$. We note that if $f_{e}\left(\sigma_{x y}\right)=1$, then $p_{x y}>1$ because $\sigma_{x y}$ consists of more than one request and a last phase containing a single request cannot be equal to the first phase. We will show that for any number $i, 1<i<p_{x y}$,

$$
O P T(\pi(i)) \geq \frac{1}{2}\left(r(\pi(i))+l_{c}(\pi(i))\right)
$$

as well as

$$
O P T(\pi(1)) \geq \frac{1}{2}\left(r(\pi(1))+l_{c}(\pi(1))\right)-f_{b}\left(\sigma_{x y}\right)
$$

and, if $p_{x y}>1$,

$$
O P T\left(\pi\left(p_{x y}\right)\right) \geq \frac{1}{2}\left(r\left(\pi\left(p_{x y}\right)\right)+l_{c}\left(\pi\left(p_{x y}\right)\right)+f_{e}\left(\sigma_{x y}\right)\right) .
$$

If $\sigma_{x y}$ consists of only one phase, then the desired inequality (2) follows from (4), taking into account that $f_{e}\left(\sigma_{x y}\right)=0$. If $\sigma_{x y}$ consists of at least two phases, then we obtain (2) by summing (3), for all $i=2, \ldots, p_{x y}-$ 1 , as well as (4) and (5).

We first analyze any phase that is not equal to the first phase and prove inequalities (3) and (5). Consider a phase $i$ with $1<i \leq p_{x y}$. Recall that the previous phase $i-1$ ended with a long run of requests to item $y$ such that $x$ is stored at position two in the list when phase $i$ starts. If $f_{e}\left(\sigma_{x y}\right)=1$, then $\pi\left(p_{x y}\right)$ consists of a single request to $x$ and (5) is easy to see: We have $l_{c}\left(\pi\left(p_{x y}\right)\right)=0$. Furthermore $O P T$ 's cost is 1 , which is equal to $\frac{1}{2}\left(r\left(\pi\left(p_{x y}\right)\right)+f_{e}\left(\sigma_{x y}\right)\right)$. Therefore we may assume $f_{e}\left(\sigma_{x y}\right)=0$ when considering phase number $p_{x y}$ and the proof inequalities (3) and (5) reduces to showing $O P T(\pi(i)) \geq \frac{1}{2}\left(r(\pi(i))+l_{c}(\pi(i))\right)$.

If phase $i$ has structure (a), then the cost incurred by $O P T$ is equal to $k+1$ because each request to $x$ in the phase prefix $(x y)^{k} x$ costs 1 . If $l \geq 2$, then $O P T$ moves $x$ to the front of the list on the first request of the long run $x^{l}$ and no further cost is incurred. The number of runs in the phase is $2 k+1$. If the phase ends with a long run, we have a long run change, i.e. $l_{c}(\pi(i))=1$. If the phase ends with a short run, i.e. $l=1$, then $l_{c}(\pi(i))=0$. We obtain $O P T(\pi(i))=k+1=\frac{1}{2}(2 k+1+1) \geq \frac{1}{2}\left(r(\pi(i))+l_{c}(\pi(i))\right)$. If phase $i$ has structure (b), then $O P T$ pays a cost of $k$ because each request to $x$ costs 1 . The number of runs in the phase is $2 k$ and $O P T(\pi(i))=k=\frac{1}{2}(2 k)=\frac{1}{2}\left(r(\pi(i))+l_{c}(\pi(i))\right)$ because no long run change occurred and hence $l_{c}(\pi(i))=0$.

We finally take care of the first phase. If $x$ is stored after $y$ in the initial list, then $f_{b}\left(\sigma_{x y}\right)=0$ and the arguments presented in the last paragraph immediately carry over. Recall that $l_{c}(\pi(1))=1$ if and only if the phase has structure (a) with $l \geq 2$. We obtain $O P T(\pi(1)) \geq \frac{1}{2}\left(r(\pi(1))+l_{c}(\pi(1))\right)=\frac{1}{2}\left(r(\pi(1))+l_{c}(\pi(1))\right)-$ $f_{b}\left(\sigma_{x y}\right)$. If $x$ is stored in front of $y$ in the initial list, then $f_{b}\left(\sigma_{x y}\right)=1$. We have $O P T(\pi(1))=\lfloor r(\pi(1)) / 2\rfloor$ because runs alternate between items $x$ and $y$. The latter expression is at least $\frac{1}{2}\left(r(\pi(1))+l_{c}(\pi(1))\right)-f_{b}\left(\sigma_{x y}\right)$ because $f_{b}\left(\sigma_{x y}\right)=1$. 


\subsection{Online algorithms}

We first study deterministic online algorithms and then address randomized strategies. The most popular online algorithm for list update is Move-To-Front.

Algorithm Move-To-Front (MTF): Move the requested item to the front of the list.

Lemma 2 The cost incurred by $M T F$ is $M T F(\sigma)=r(\sigma)-f_{b}(\sigma)+|\sigma|$.

Proof. We first argue that $M T F_{x y}(\sigma)=M T F\left(\sigma_{x y}\right)$ holds for any item pair $x, y$ with $x \neq y$. When $M T F$ serves a request sequence, at any time item $x$ precedes item $y$ in the current list if and only if the last request made to an item from $\{x, y\}$ was to $x$ rather than to $y$. This holds when $M T F$ serves $\sigma$ on the entire list consisting of all the items in $L$ as well as when $M T F$ serves $\sigma_{x y}$ on the two-item list consisting of $x$ and $y$ only. Sequence $\sigma$ consists of $\left|\sigma_{x y}\right|$ requests to $x$ and $y$. Thus, for any $i$ with $1 \leq i \leq\left|\sigma_{x y}\right|$, the $i$ th request made to an item from $\{x, y\}$ in $\sigma$ contributes 1 in $M T F_{x y}(\sigma)$ if and only if the $i$ th request in $\sigma_{x y}$ incurs a cost of 1 in $M T F\left(\sigma_{x y}\right)$. Note that $M T F$ does not use paid exchanges. We conclude, as desired $M T F_{x y}(\sigma)=\operatorname{MTF}\left(\sigma_{x y}\right)$.

Hence, using (1), we find $M T F(\sigma)=\sum_{\{x, y\} \subseteq L, x \neq y} M T F\left(\sigma_{x y}\right)+|\sigma|$. Consider a fixed pair of items $x, y \in L$ with $x \neq y$. We prove $M T F\left(\sigma_{x y}\right)=r\left(\sigma_{x y}\right)-f_{b}\left(\sigma_{x y}\right)$. Summing this equation over all pairs of items and using $M T F(\sigma)=\sum_{\{x, y\} \subseteq L, x \neq y} M T F\left(\sigma_{x y}\right)+|\sigma|$, we obtain the lemma. When $M T F$ serves $\sigma_{x y}$ on the two-item list, on the first request of each run the referenced item is moved to the front of the list. Hence on each run, except for possibly the first one, $M T F$ incurs a cost of exactly 1 . On the first run, the cost is 1 if the requested item is stored behind the other item of $\{x, y\}$ in the initial list. The cost is 0 if the requested item precedes the other item of $\{x, y\}$ in the initial list and in this case $f_{b}\left(\sigma_{x y}\right)=1$. These arguments yield the equation to be proven.

For any request sequence $\sigma$, let $\alpha(\sigma)=\left(|\sigma|-f_{b}(\sigma)\right) / r(\sigma)$. Furthermore, let $\beta(\sigma)=l_{c}(\sigma) / r(\sigma)$ be the fraction of the long run changes relative to the total number of runs. The following theorem gives a refined bound on the performance ratio of $M T F$. It implies, in particular, that $M T F$ is 2-competitive.

Theorem 1 For any request sequence $\sigma$, the cost incurred by MTF is at most $\frac{2+2 \alpha(\sigma)}{1+2 \alpha(\sigma)+\beta(\sigma)}$ times that payed by $O P T$.

Proof. Applying Lemmas 1 and 2 we find that the ratio of the cost incurred by $M T F$ to that payed by $O P T$ is upper bounded by

$$
\begin{aligned}
c & =\frac{r(\sigma)-f_{b}(\sigma)+|\sigma|}{\frac{1}{2}\left(r(\sigma)+l_{c}(\sigma)+f_{e}(\sigma)\right)-f_{b}(\sigma)+|\sigma|} \leq \frac{r(\sigma)-f_{b}(\sigma)+|\sigma|}{\frac{1}{2}\left(r(\sigma)+l_{c}(\sigma)\right)-f_{b}(\sigma)+|\sigma|} \\
& =\frac{2+2\left(|\sigma|-f_{b}(\sigma)\right) / r(\sigma)}{1+l_{c}(\sigma) / r(\sigma)+2\left(|\sigma|-f_{b}(\sigma)\right) / r(\sigma)}=\frac{2+2 \alpha(\sigma)}{1+2 \alpha(\sigma)+\beta(\sigma)} .
\end{aligned}
$$

An immediate consequence of the above theorem is the following corollary. It implies that $M T F$ can achieve a competitiveness as low as 1 on request sequences that exhibit a high degree of locality, i.e. that satisfy $\lambda$-locality with values of $\lambda$ close to 1 .

Corollary 1 On request sequences exhibiting $\lambda$-locality, MTF achieves a competitive ratio of at most $\frac{2}{1+\lambda}$. 
A second important deterministic online strategy is Timestamp. The algorithm is used, in particular, to construct the best randomized online strategy currently known.

Algorithm Timestamp (TS): Insert the requested item, say $x$, immediately in front of the first item in the list that precedes $x$ in the current list and was requested at most once since the last request to $x$. If there is no such item or if $x$ is requested for the first time, do not change the position of $x$.

Lemma 3 The cost incurred by $T S$ is $T S(\sigma) \leq r(\sigma)+l_{i}(\sigma)-l_{p}(\sigma)+f_{b}^{\prime}(\sigma)+|\sigma|$.

Proof. Again, we first argue that $T S_{x y}(\sigma)=T S\left(\sigma_{x y}\right)$ for any item pair $x, y$ with $x \neq y$. In the following paragraph we will show that when $T S$ serves a request sequence $\sigma$ on the full list, the referenced item, say $x$, never passes an item in the list that was requested at least twice since the last request to $x$. Thus the following property holds: When $T S$ serves the $i$ th request to $x$ or $y$ in $\sigma$, the relative order of the two items in the full list is the same as that in the two-item list consisting of $x$ and $y$ when $T S$ serves the $i$ th request in $\sigma_{x y}$, $1 \leq i \leq\left|\sigma_{x y}\right|$. We conclude $T S_{x y}(\sigma)=T S\left(\sigma_{x y}\right)$.

It remains to show that when $T S$ serves a request sequence $\sigma$ on the full list, the referenced item, say $x$, never passes an item in the list that was requested at least twice since the last request to $x$. We prove the statement by induction on $t$. Consider an arbitrary request sequence $\sigma$. The statement holds at time $t=1$ because the referenced item $\sigma(1)$ is requested for the first time and $T S$ does not change its position in the list. Suppose that the desired statement holds up to time $t-1$, and let $x=\sigma(t)$ be the item referenced at time $t$. If $x$ is requested for the first time, then again the position of $x$ in the list does not change. Otherwise let $y$ be the first item in the current list that precedes $x$ and was requested at most once since the last request to $x$. (In case no such $y$ exists, the position of $x$ remains unchanged.) Let $z$ be any item that was requested at least twice since the last request to $x$. The last three requests made to either $y$ or $z$ are of the following form: (a) $z \ldots z \ldots y$; (b) $z \ldots y \ldots z$ or (c) $y \ldots z \ldots z$. In each case $z$ precedes $y$ in the current list after the above subsequence of requests. In case (a) this follows from induction hypothesis. In cases (b) and (c) this holds because on requests to $z$ this item passes all items that were referenced at most once since the last request to $z$. We conclude that when $x$ is inserted in front of $y$, item $z$ is not passed.

Thus, using (1), we obtain

$$
T S(\sigma)=\sum_{\substack{\{x, y\} \subseteq L \\ x \neq y}} T S\left(\sigma_{x y}\right)+|\sigma| .
$$

We consider a fixed pair of items $x, y \in L$ with $x \neq y$ and will prove

$$
T S\left(\sigma_{x y}\right) \leq s\left(\sigma_{x y}\right)+2 l_{i}\left(\sigma_{x y}\right)+f_{b}^{\prime}\left(\sigma_{x y}\right) .
$$

As $r\left(\sigma_{x y}\right)=s\left(\sigma_{x y}\right)+l\left(\sigma_{x y}\right)=s\left(\sigma_{x y}\right)+l_{p}\left(\sigma_{x y}\right)+l_{i}\left(\sigma_{x y}\right)$ we find $T S\left(\sigma_{x y}\right) \leq r\left(\sigma_{x y}\right)+l_{i}\left(\sigma_{x y}\right)-l_{p}\left(\sigma_{x y}\right)+$ $f_{b}^{\prime}\left(\sigma_{x y}\right)$. Summing the latter inequality over all pairs of items, taking into account (6), we obtain the lemma.

For the analysis of $T S\left(\sigma_{x y}\right)$ we partition $\sigma_{x y}$ again into phases as described in Section 3.1. We consider an arbitrary phase $\pi(i), 1 \leq i \leq p_{x y}$, and assume w.l.o.g. that the phase starts with a request to item $x$. Recall that phase $\pi(i)$ has one of the following two structures.

$$
\begin{array}{ll}
\text { (a) }(x y)^{k} x^{l} \quad k \geq 0, l \geq 1 & \text { (b) }(x y)^{k} y^{l} \quad k \geq 1, l \geq 0
\end{array}
$$

The independent long runs in $\sigma_{x y}$ are exactly the phases of structure (a) with $k=0$ and $l \geq 2$. When $T S$ serves $\sigma_{x y}$ on the two-item list, on the second request of each long run the algorithm moves the referenced item to the front of the list if it is not already there. Hence on each long run TS pays a cost of at most 2. This proves that on all independent long runs, i.e. on all phases of structure (a) with $k=0$ and $l \geq 2, T S$ 's total cost is upper bounded by $2 l_{i}\left(\sigma_{x y}\right)$. In the following we prove that on all other phases, $T S$ incurs a cost of at 
most $s(\pi(i))$, where $s(\pi(i))$ denotes the number of short runs in $\pi(i)$. In the first phase the cost is by 1 larger if $f_{b}^{\prime}\left(\sigma_{x y}\right)=1$.

Consider an arbitrary phase $\pi(i)$ and suppose first that $i>1$. At the beginning of the phase item $x$ is stored behind $y$ in the list because the last two requests were made to $y$. Thus $T S$ incurs a cost of 1 on the first request of the phase. If the phase consists of only one request, we are done because in this case the number of short runs is also 1. Therefore assume that the phase consists of at least two requests. When serving the first request of $\pi(i), T S$ leaves $x$ 's position in the list unchanged because $y$ was requested at least twice since the last reference to $x$. Hence the second request of the phase, which is to $y$, does not incur cost and we have identified a short run that does not incur cost. We will use this fact in the next paragraph. If the phase is composed of short runs only, we are done because each further short run can incur a cost of at most 1; in fact the cost is exactly 1 .

If the phase ends with a long run, then in phase structure (a) we have $k \geq 1$ because $k=0$ would imply that we deal with an independent long run, which was already analyzed above. Hence, on the first request of the long run $x^{l} T S$ moves $x$ to the front of the list so that the run incurs a cost of only 1 . Recall that the second short run of the phase does not incur any cost. Hence the cost paid by $T S$ in $\pi(i)$ is exactly equal to the number $s(\pi(i))$ of short runs. If the phase ends with a long run and has structure (b), then the suffix $y^{l}$ does not incur cost: This holds true if $k=1$ because, as argued above, $x$ is not moved in front of $y$ on the first request of the phase. If $k>1$, then on the last request of $(x y)^{k} T S$ moves $y$ in front of $x$ in the list because $x$ was referenced at most once since the last request to $x$. Hence $T S$ pays a cost of $2 k-1$ in the phase, which is equal to the number of short runs.

We finally study the first phase $\pi(1)$. The arguments presented in the previous paragraph immediately carry over if $x$ is stored behind $y$ in the initial list because on the first request to $x$ algorithm $T S$ does not change the position of $x$ in the list and a second request of the phase, which is to $y$, does not incur cost. Therefore assume that $x$ is stored in front of $y$ in the initial list. In this case the first run of the phase does not incur cost, and we have again identified a run not causing any cost. If the phase is composed of short runs only, we are done because the service cost of $T S$ in $\pi(1)$ is upper bounded by $s(\pi(1))$. Therefore suppose that the phase ends with a long run. This implies that the phase consists of at least two runs because a single long run would represent an independent long run, whose cost was studied before. If the phase is composed of two runs, then its structure is $x y^{l^{\prime}}$, for some $l^{\prime} \geq 2$. TS pays a cost of 2 in the phase because on the first request to $y$ it does not change the position of $y$ in the list as the item is referenced for the first time. On the second request to $y$, the item is moved to the front of the list and incurs no further cost in the phase. This cost of 2 is equal to the number of short runs plus $f_{b}^{\prime}\left(\sigma_{x y}\right)$.

If the phase is composed of at least three runs, the item referenced in the final long run $\rho$ was requested at least once before and hence moves to the front of the list after the first request of $\rho$, if not already there, because the other item of the pair was requested only once in between. Thus the final long run incurs a cost of at most 1 . Recalling that the first run of $\pi(1)$ does not generate cost, we conclude that $T S$ 's total cost in $\pi(1)$ is bounded by the number of short runs of the phase.

We observe that $T S$ is better than $M T F$ if the number of prefixed long runs is larger than the number of independent long runs plus $f_{b}^{\prime}(\sigma)$.

Recall that $\alpha(\sigma)=\left(|\sigma|-f_{b}(\sigma)\right) / r(\sigma)$ and $\beta(\sigma)=l_{c}(\sigma) / r(\sigma)$. Furthermore, let $\alpha^{\prime}(\sigma)=\left(f_{b}(\sigma)+\right.$ $\left.f_{b}^{\prime}(\sigma)\right) / r(\sigma)$ and $\gamma(\sigma)=\left(l_{i}(\sigma)-l_{p}(\sigma)\right) / r(\sigma)$.

Theorem 2 For any request sequence $\sigma$, the cost incurred by TS is at most $\frac{2+2 \alpha(\sigma)+2 \alpha^{\prime}(\sigma)+2 \gamma(\sigma)}{1+2 \alpha(\sigma)+\beta(\sigma)}$ times that payed by $O P T$.

Proof. Applying Lemmas 1 and 3 we find that the ratio of the cost incurred by $T S$ to that payed by $O P T$ is 
upper bounded by

$$
\begin{aligned}
c & =\frac{r(\sigma)+l_{i}(\sigma)-l_{p}(\sigma)+f_{b}^{\prime}(\sigma)+|\sigma|}{\frac{1}{2}\left(r(\sigma)+l_{c}(\sigma)+f_{e}(\sigma)\right)-f_{b}(\sigma)+|\sigma|} \leq \frac{r(\sigma)+l_{i}(\sigma)-l_{p}(\sigma)+f_{b}^{\prime}(\sigma)+|\sigma|}{\frac{1}{2}\left(r(\sigma)+l_{c}(\sigma)\right)-f_{b}(\sigma)+|\sigma|} \\
& \leq \frac{r(\sigma)+l_{i}(\sigma)-l_{p}(\sigma)+f_{b}(\sigma)+f_{b}^{\prime}(\sigma)+|\sigma|-f_{b}(\sigma)}{\frac{1}{2}\left(r(\sigma)+l_{c}(\sigma)\right)+|\sigma|-f_{b}(\sigma)} \\
& =\frac{2+2\left(l_{i}(\sigma)-l_{p}(\sigma)\right) / r(\sigma)+2\left(f_{b}(\sigma)+f_{b}^{\prime}(\sigma)\right) / r(\sigma)+2\left(|\sigma|-f_{b}(\sigma)\right) / r(\sigma)}{1+l_{c}(\sigma) / r(\sigma)+2\left(|\sigma|-f_{b}(\sigma)\right) / r(\sigma)} \\
& =\frac{2+2 \alpha(\sigma)+2 \alpha^{\prime}(\sigma)+2 \gamma(\sigma)}{1+2 \alpha(\sigma)+\beta(\sigma)} .
\end{aligned}
$$

We have $l_{i}(\sigma) \leq l_{c}(\sigma)$ and $f_{b}^{\prime}(\sigma) \leq f_{b}(\sigma) \leq|L|(|L|-1) / 2$. Therefore, $\gamma(\sigma) \leq \beta(\sigma)$ and $\alpha^{\prime}(\sigma)$ is upper bounded by a constant that is independent of $\sigma$. Hence the above theorem also yields that TS is 2-competitive. On the other hand, $l_{p}(\sigma)$ can be 0 and $l_{i}(\sigma)$ can be as high as $l_{c}(\sigma)$. In this case $\gamma(\sigma)=\beta(\sigma)$. Thus our refined analysis does not yield an improved competitive ratio for $T S$ on request sequences satisfying $\lambda$-locality.

We next turn to randomized algorithms and consider the popular Bit strategy.

Algorithm BIT: Maintain a bit $b(x)$ for each item $x \in L$. These bits are initialized independently and uniformly at random to a value in $\{0,1\}$. On a request, complement the bit of the referenced item. If the bit value changes to 1 , move the item to the front of the list.

For the proof of Lemma 4 below we need the following claim.

Claim 1 Assume that BIT serves $\sigma_{x y}$ on a list consisting of $x$ and $y$ only.

(1) Suppose that $x$ is stored after $y$ in the two-item list and that $x$ is requested. Then the expected cost of the following request is equal to $1 / 2$.

(2) Suppose that BIT has just served a subsequence xyx of requests. If the next request is to $y$, then BIT's expected cost on that request is equal to $3 / 4$. If the next request is to $x$, the expected cost is equal to $1 / 4$.

Analogous statements hold if the roles of $x$ and $y$ are interchanged.

Proof. Consider any item $x$. After $B I T$ has served $i$ requests to $x$, the bit $b(x)$ has value $\left(b_{0}(x)+i\right) \bmod 2$, where $b_{0}(x)$ is the initial bit value. Thus, whenever $B I T$ serves a request to $x$, the value of $b(x)$ is equally likely to be 0 or 1 . This was also shown in [35].

To verify part (1) of the claim we simply observe that when serving the request to $x$, with probability $1 / 2$ $B I T$ moves $x$ to the front of the list. Thus, after the request, $x$ precedes $y$ in the list with probability exactly $1 / 2$. To verify part (2) we remark that after the service of the subsequence $x y x$, item $x$ precedes $y$ in the list if and only if (a) $x$ is moved to the front of the list on the second request to $x$ or if (b) $x$ is not moved to the front of the list on the second request to $x$ (such that it was moved to the front on the first request to $x$ ) and $y$ is not moved to the front on its reference in $x y x$. Event (a) occurs with probability $1 / 2$ while (b) occurs with probability $1 / 4$.

Lemma 4 The expected cost incurred by BIT is BIT $(\sigma) \leq \frac{3}{4} r(\sigma)+\frac{1}{4} l(\sigma)+\frac{1}{2} l_{i}(\sigma)+\frac{1}{4} f_{e}(\sigma)+|\sigma|$.

Proof. The question whether or not BIT moves a requested item, say $x$, to the front of the list only depends on the initial bit value $b_{0}(x)$ and the number of requests to $x$ processed so far. On the $i$ th request to $x$, the item 
is moved to the front of the list if $\left(b_{0}(x)+i\right) \bmod 2=1$. Thus when $B I T$ serves the $i$ th request to either $x$ or $y$ in $\sigma$, item $x$ precedes $y$ in the full list if and only if $x$ precedes $y$ in the two-item list when $B I T$ serves the $i$ th request in $\sigma_{x y}, 1 \leq i \leq\left|\sigma_{x y}\right|$. Hence $B I T_{x y}(\sigma)=B I T\left(\sigma_{x y}\right)$, for any pair $x, y \in L$ with $x \neq y$, and we obtain

$$
B I T(\sigma)=\sum_{\substack{\{x, y\} \subseteq L \\ x \neq y}} B I T\left(\sigma_{x y}\right)+|\sigma| .
$$

Fix an item pair $x, y \in L$ with $x \neq y$. We will prove that the expected cost incurred by BIT is

$$
B I T\left(\sigma_{x y}\right) \leq \frac{3}{4} r\left(\sigma_{x y}\right)+\frac{1}{4} l\left(\sigma_{x y}\right)+\frac{1}{2} l_{i}\left(\sigma_{x y}\right)+\frac{1}{4} f_{e}\left(\sigma_{x y}\right) .
$$

We obtain the lemma by summing the last inequality over all item pairs, taking into account (8).

We evaluate $B I T\left(\sigma_{x y}\right)$ by partitioning, as usual, $\sigma_{x y}$ into phases as described in Section 3.1. Consider an arbitrary phase $\pi(i), 1 \leq i \leq p_{x y}$, and assume w.l.o.g. that the phase starts with a request to item $x$. Phase $\pi(i)$ has one of the following two structures.

$$
\begin{array}{ll}
\text { (a) }(x y)^{k} x^{l} \quad k \geq 0, l \geq 1 & \text { (b) }(x y)^{k} y^{l} \quad k \geq 1, l \geq 0
\end{array}
$$

If $\sigma_{x y}$ consists of a single request to item, say $x$, we can easily verify (9): If $x$ is stored in front of $y$ in the initial list, then the cost incurred by BIT is 0 and the right hand side of (9) is lower bounded by 0 . On the other hand, if $x$ is stored after $y$ in the initial list, then the cost incurred by BIT is 1 . The right hand side of (9) is also equal to 1 because $r\left(\sigma_{x y}\right)=1$ and $f_{e}\left(\sigma_{x y}\right)=1$ while the other terms are 0 .

In the remainder of this proof we assume that $\sigma_{x y}$ consists of more than one request and analyze the various phases. For any $i$ with $1 \leq i \leq p_{x y}$, let $r(\pi(i))$ be the number of runs in $\pi(i)$. Furthermore, let $l(\pi(i))$ be equal to 1 if the phase contains a long run; otherwise $l(\pi(i))=0$. Finally, we set $l_{i}(\pi(i))$ equal to 1 if the phase consists of an independent long run; otherwise $l_{i}(\pi(i))=0$. Let $B I T(\pi(i))$ denote the expected cost incurred by $B I T$ in the phase. Note that if $f_{e}\left(\sigma_{x y}\right)=1$, then $p_{x y}>1$ because the request sequence consists of more than one request and the first phase cannot consist of a single request. We will show that for any phase number $i$ satisfying $1 \leq i<p_{x y}$,

$$
B I T(\pi(i)) \leq \frac{3}{4} r(\pi(i))+\frac{1}{4} l(\pi(i))+\frac{1}{2} l_{i}(\pi(i))
$$

and for index $p_{x y}$,

$$
B I T\left(\pi\left(p_{x y}\right)\right) \leq \frac{3}{4} r\left(\pi\left(p_{x y}\right)\right)+\frac{1}{4} l\left(\pi\left(p_{x y}\right)\right)+\frac{1}{2} l_{i}\left(\pi\left(p_{x y}\right)\right)+\frac{1}{4} f_{e}\left(\sigma_{x y}\right) .
$$

If $\sigma_{x y}$ consists of only one phase, then the desired inequality (9) follows from (11). If $\sigma_{x y}$ consists of at least two phases, we derive (9) by summing (10), for all $i=1, \ldots, p_{x y}-1$, and (11).

If $i=p_{x y}$ and $f_{e}\left(\sigma_{x y}\right)=1$, then (11) follows easily: Phase $\pi\left(p_{x y}\right)$ consists of a single request to an item, say $x$. The cost incurred by $B I T$ is 1 , and this is equal to $\frac{3}{4} r\left(\pi\left(p_{x y}\right)\right)+\frac{1}{4} f_{e}\left(\sigma_{x y}\right)$. Inequality (11) follows because $l\left(\pi\left(p_{x y}\right)\right)=l_{i}\left(\pi\left(p_{x y}\right)\right)=0$. Therefore, when analyzing the last phase indexed $i=p_{x y}$, we may assume $f_{e}\left(\sigma_{x y}\right)=0$ and proving (10) and (11) reduced to showing

$$
B I T(\pi(i)) \leq \frac{3}{4} r(\pi(i))+\frac{1}{4} l(\pi(i))+\frac{1}{2} l_{i}(\pi(i))
$$

for any $i$ with $1 \leq i \leq p_{x y}$. We first analyze a phase $\pi(i)$, where either (a) $i \geq 2$ or (b) $i=1$ and $f_{b}\left(\sigma_{x y}\right)=0$ hold. Then we study the remaining case that $i=1$ and $f_{b}\left(\sigma_{x y}\right)=1$.

So consider a phase $\pi(i)$, where either $i \geq 2$ or $i=1$ combined with $f_{b}\left(\sigma_{x y}\right)=0$ hold. We first argue that when $\pi(i)$ starts, $x$ is stored behind $y$ in the list. This obviously holds if $i=1$ because $f_{b}\left(\sigma_{x y}\right)=0$ 
indicates that the item first requested in $\sigma_{x y}$ and hence in the first phase is stored after the other item in the initial list. If $i \geq 2$, then phase $\pi(i)$ is preceded by a long run of requests to $y$. After the service of the second request of this long run, $y$ definitely precedes $x$ in the list. Hence the first request of $\pi(i)$ incurs a cost of 1 and using Claim 1, part (1) we obtain that the second request of the phase costs $1 / 2$. If the phase has structure (a) with $k=0$, the total cost of the phase is $3 / 2$, which is equal to $\frac{3}{4} r(\pi(i))+\frac{1}{4} l(\pi(i))+\frac{1}{2} l_{i}(\pi(i))$ because $r(\pi(i))=l(\pi(i))=l_{i}(\pi(i))=1$ as $\pi(i)$ represents an independent long run. Inequality (12) holds.

Therefore we may assume $k \geq 1$ in both structure (a) and (b), which implies $l_{i}(\pi(i))=0$. Again, the first two requests $x y$ in the phase cause a total cost of $3 / 2$. Using Claim 1, part (2) we find that BIT pays an expected cost of $3 / 4$ for the service of any further short run in the phase. If the phase consists of only short runs, then $B I T$ 's expected cost is $\frac{3}{4} r(\pi(i))$ and (12) holds because $l(\pi(i))=0$. So suppose that $\pi(i)$ ends with a long run. If the phase has structure (a), then Claim 1, part (2) implies that the first two requests of the suffix $x^{l}$ incur a cost of $3 / 4+1 / 4$. On the second request of this long run $x$ is moved to the front of the list, if $x$ is not already there, and the run incurs no further cost. Thus $B I T(\pi(i))=\frac{3}{2} k+1=\frac{3}{4} r(\pi(i))+\frac{1}{4} l(\pi(i))$ because $l(\pi(i))=1$. Inequality (12) holds. If the phase has structure (b), then Claim 1, part (2) with the roles of $x$ and $y$ interchanged implies that the first request of $y^{l}$ incurs an expected cost of $1 / 4$. As $y$ is moved to the front of the list, if not already there, the run incurs no further cost. We obtain $B I T(\pi(i))=\frac{3}{2} k+\frac{1}{4}=\frac{3}{4} r(\pi(i))+\frac{1}{4} l(\pi(i))$ because, again, $l(\pi(i))=1$.

We finally have to study the first phase $\pi(1)$ if $f_{b}\left(\sigma_{x y}\right)=1$, i.e. the item first requested in $\pi(1)$ precedes the other item of the pair in the initial list. In this case the first request of the phase incurs a cost of 0 . If $\pi(1)$ consists of a single long run, then BIT's total cost in the phase is 0 . Inequality (12) holds because the right-hand side is lower bounded by 0 . So assume that $\pi(1)$ consists of at least two runs, which implies $l_{i}(\pi(1))=0$. In this case the second request of the phase, which is to $y$, incurs a cost of 1 . If the phase consists of exactly two runs, then $\pi(1)=x y^{l}$ where $l \geq 1$, and using Claim 1, part (1) we obtain that BIT's expected cost is at most $3 / 2$, which in turn is upper bounded by $\frac{3}{4} r(\pi(1))+\frac{1}{4} l(\pi(1))$ because $r(\pi(1))=2$ and $l(\pi(1))=1$. We finally focus on the case that $\pi(1)$ is composed of at least three runs. In this case we can simply consider a truncated phase $\pi^{\prime}(1)$ derived from $\pi(1)$ by removing the first request. In this truncated phase a final long run is still preceded by a short run and the item first requested in $\pi^{\prime}(1)$ precedes the other item of the pair when the phase starts. Applying all the arguments of the previous paragraph we obtain (12), taking into account that the original phase $\pi(1)$ contains even one run more than $\pi^{\prime}(1)$.

$$
\text { Again, } \alpha(\sigma)=\left(|\sigma|-f_{b}(\sigma)\right) / r(\sigma) \text { and } \beta(\sigma)=l_{c}(\sigma) / r(\sigma) \text {. Define } \delta(\sigma)=\left(\frac{1}{2} l(\sigma)+l_{i}(\sigma)+2 f_{b}(\sigma)\right) / r(\sigma) \text {. }
$$

Theorem 3 For any request sequence $\sigma$, the expected cost incurred by BIT is at most $\frac{1.5+2 \alpha(\sigma)+\delta(\sigma)}{1+2 \alpha(\sigma)+\beta(\sigma)}$ times that payed by $O P T$.

Proof. Applying Lemmas 1 and 4 we find that the ratio of the expected cost incurred by BIT to that payed by $O P T$ is upper bounded by

$$
\begin{aligned}
c & =\frac{\frac{3}{4} r(\sigma)+\frac{1}{4} l(\sigma)+\frac{1}{2} l_{i}(\sigma)+\frac{1}{4} f_{e}(\sigma)+|\sigma|}{\frac{1}{2}\left(r(\sigma)+l_{c}(\sigma)+f_{e}(\sigma)\right)-f_{b}(\sigma)+|\sigma|} \\
& \leq \frac{\frac{3}{4} r(\sigma)+\frac{1}{4} l(\sigma)+\frac{1}{2} l_{i}(\sigma)+|\sigma|}{\frac{1}{2}\left(r(\sigma)+l_{c}(\sigma)\right)-f_{b}(\sigma)+|\sigma|} \\
& \leq \frac{1.5 r(\sigma)+\frac{1}{2} l(\sigma)+l_{i}(\sigma)+2 f_{b}(\sigma)+2\left(|\sigma|-f_{b}(\sigma)\right)}{r(\sigma)+l_{c}(\sigma)+2\left(|\sigma|-f_{b}(\sigma)\right)} \\
& =\frac{1.5+\left(\frac{1}{2} l(\sigma)+l_{i}(\sigma)+2 f_{b}(\sigma)\right) / r(\sigma)+2\left(|\sigma|-f_{b}(\sigma)\right) / r(\sigma)}{1+l_{c}(\sigma) / r(\sigma)+2\left(|\sigma|-f_{b}(\sigma)\right) / r(\sigma)} \\
& =\frac{1.5+2 \alpha(\sigma)+\delta(\sigma)}{1+2 \alpha(\sigma)+\beta(\sigma)} .
\end{aligned}
$$


It is not hard to show that the above theorem also implies that BIT is 1.75 -competitive. Taking into account that $l(\sigma) \leq r(\sigma)$ and $l_{i}(\sigma) \leq l_{c}(\sigma)$, we obtain the following corollary, which yields that BIT attains a competitiveness of 1.5 on request sequences with a high degree of locality, i.e. with values of $\lambda$ close to 1 .

Corollary 2 On request sequences exhibiting $\lambda$-locality, BIT achieves a competitive ratio of $\min \left\{1.75, \frac{2+\lambda}{1+\lambda}\right\}$.

We finally turn to $C O M B$, the list update algorithm achieving the smallest competitive ratio currently known.

Algorithm Combination (COMB): With probability $4 / 5$ serve the request sequence using $B I T$, and with probability $1 / 5$ serve the sequence using $T S$.

Lemma 5 The expected cost incurred by $C O M B$ is $C O M B(\sigma) \leq \frac{1}{5}\left(4 r(\sigma)+4 l_{i}(\sigma)+f_{e}(\sigma)+f_{b}^{\prime}(\sigma)\right)+|\sigma|$.

Proof. By the definition of $C O M B$, on any request sequence, the algorithm's expected cost is $\frac{4}{5} B I T(\sigma)+$ $\frac{1}{5} T S(\sigma)$ such that, using Lemmas 3 and 4 , we obtain

$$
\begin{aligned}
C O M B(\sigma) & \leq \frac{1}{5}\left(3 r(\sigma)+l(\sigma)+2 l_{i}(\sigma)+f_{e}(\sigma)\right)+\frac{1}{5}\left(r(\sigma)+l_{i}(\sigma)-l_{p}(\sigma)+f_{b}^{\prime}(\sigma)\right)+|\sigma| \\
& =\frac{1}{5}\left(4 r(\sigma)+l(\sigma)+3 l_{i}(\sigma)-l_{p}(\sigma)+f_{e}(\sigma)+f_{b}^{\prime}(\sigma)\right)+|\sigma| \\
& =\frac{1}{5}\left(4 r(\sigma)+4 l_{i}(\sigma)+f_{e}(\sigma)+f_{b}^{\prime}(\sigma)\right)+|\sigma| .
\end{aligned}
$$

The last line follows because $l(\sigma)=l_{i}(\sigma)+l_{p}(\sigma)$.

$$
\text { Let } \zeta(\sigma)=\left(l_{i}(\sigma)+\frac{5}{4} f_{b}(\sigma)+\frac{1}{4} f_{b}^{\prime}(\sigma)\right) / r(\sigma) .
$$

Theorem 4 For any request sequence $\sigma$, the expected cost incurred by COMB is at most $\frac{1.6+2 \alpha(\sigma)+1.6 \zeta(\sigma)}{1+2 \alpha(\sigma)+\beta(\sigma)}$ times that payed by OPT.

Proof. Using Lemmas 1 and 4 we obtain that the ratio of the expected cost of $C O M B$ to that of $O P T$ is upper bounded by

$$
\begin{aligned}
c & =\frac{\frac{1}{5}\left(4 r(\sigma)+4 l_{i}(\sigma)+f_{e}(\sigma)+f_{b}^{\prime}(\sigma)\right)+|\sigma|}{\frac{1}{2}\left(r(\sigma)+l_{c}(\sigma)+f_{e}(\sigma)\right)-f_{b}(\sigma)+|\sigma|} \\
& \leq \frac{\frac{1}{5}\left(4 r(\sigma)+4 l_{i}(\sigma)+f_{b}^{\prime}(\sigma)\right)+|\sigma|}{\frac{1}{2}\left(r(\sigma)+l_{c}(\sigma)\right)-f_{b}(\sigma)+|\sigma|} \\
& \leq \frac{1.6 r(\sigma)+1.6 l_{i}(\sigma)+0.4 f_{b}^{\prime}(\sigma)+2|\sigma|}{r(\sigma)+l_{c}(\sigma)-2 f_{b}(\sigma)+2|\sigma|} \\
& =\frac{1.6+1.6\left(l_{i}(\sigma)+\frac{5}{4} f_{b}(\sigma)+\frac{1}{4} f_{b}^{\prime}(\sigma)\right) / r(\sigma)+2\left(|\sigma|-f_{b}(\sigma)\right) / r\left(\sigma_{x y}\right)}{1+l_{c}(\sigma) / r(\sigma)+2\left(|\sigma|-f_{b}(\sigma)\right) / r\left(\sigma_{x y}\right)} \\
& =\frac{1.6+2 \alpha(\sigma)+1.6 \zeta(\sigma)}{1+2 \alpha(\sigma)+\beta(\sigma)} .
\end{aligned}
$$

We have $l_{i}(\sigma) \leq l_{c}(\sigma)$ and $f_{b}^{\prime}(\sigma) \leq f_{b}(\sigma) \leq|L|(|L|-1) / 2$. Hence the above theorem also implies that $C O M B$ is 1.6 -competitive. Since $l_{i}(\sigma)$ can be as high as $l_{c}(\sigma)$, Theorem 4 does not give an improved competitive ratio for $C O M B$ on request sequences satisfying $\lambda$-locality. 


\section{Experimental study}

In this section we report on the results of our experimental study in which we have implemented the algorithms analyzed in Section 3. The main purpose of our study is to compare the experimentally observed performance of the algorithms to the bounds stated in Theorems $1-4$ as well as Corollaries 1 and 2. In order to get meaningful results we have tested the algorithms on real-world request sequences from benchmark libraries. Clearly, self-organizing linear lists represent a solution to the classic dictionary problem, where we have to maintain a set of elements so as to efficiently perform search and possible update operations. When a dictionary is maintained by a computer/CPU, requests are actually memory accesses. A second important application of self-organizing linear lists is data compression, where we wish to store a file using few bits. A first, general finding is that the experimental results are consistent for both data sets. In fact, the results are even slightly more positive for the memory access traces.

Since data compression has gained considerable popularity and importance, as far as the application of list update algorithms is concerned, we report on the corresponding results first and then present the results for the memory access strings.

\subsection{Data compression}

Bentley et al. [13] showed that self-organizing linear lists can be used to build locally adaptive data compression schemes. The approach was further developed and studied in $[2,16,28]$. The best compression results are achieved when the scheme is combined with the famous Burrows-Wheeler transformation [16], yielding compression rates that are comparable or even better than that of Lempel-Ziv based schemes. In fact the common open source data compression program bzip2 consists of a Burrows-Wheeler transformation followed by Move-To-Front and Huffman encodings. We briefly describe the basic data compression scheme by Bentley et al. [13] and the Burrow-Wheeler transformation [16].

Data compression schemes: In data compression we are given a string $S$ that shall be compressed, i.e. that shall be represented using fewer bits. The string $S$ consists of symbols, where each symbol is element of an alphabet $X=\left\{x_{1}, \ldots, x_{n}\right\}$. The idea of data compression schemes using linear lists is to convert the string $S$ of symbols into a string $I$ of integers. An encoder maintains a linear list of symbols contained in $X$ and reads the symbols in the string $S$. Whenever the symbol $x_{i}$ has to be compressed, the encoder looks up the current position of $x_{i}$ in the linear list, outputs this position and updates the list using a list update algorithm. Here one can use $M T F$ or any other list update strategy. If symbols to be compressed are moved closer to the front of the list, then frequently occurring symbols can be encoded with small integers. Clearly, when the string $I$ is actually stored or transmitted, each integer in the string should be coded again using a variable length prefix code.

The refined compression scheme by Burrows and Wheeler first applies a transformation to the string $S$. The purpose of this transformation is to group together instances of a symbol $x_{i}$ occurring in $S$ so that the resulting string $S^{\prime}$ exhibits a high degree of locality of reference. Of course the transformation is reversible so that, given $S^{\prime}$, the original string can be recovered. We refer the reader to [16] for details on the BurrowsWheeler transformation and its efficient implementation. The transformed string $S^{\prime}$ is then encoded using the algorithm by Bentley et al. [13] as described in the previous paragraph. Alphabet $X$, i.e. the set of entries in the self-organizing linear list, is the ASCII alphabet with its 256 different characters. The initial list is given by the initial numerical order of the ASCII characters. In the string $S$, each byte represents a symbol. For large files to be compressed, the Burrows-Wheeler transformation is applied not to the entire file but rather to blocks of uniform size; the block size may be chosen by a user.

Data sets and their locality characteristics: In our experiments we selected files available at the repository named Canterbury Corpus [17]. This collection was developed as an extension of the widely-used Calgary Corpus and represents the standard benchmark library for evaluating data compression algorithms. It 


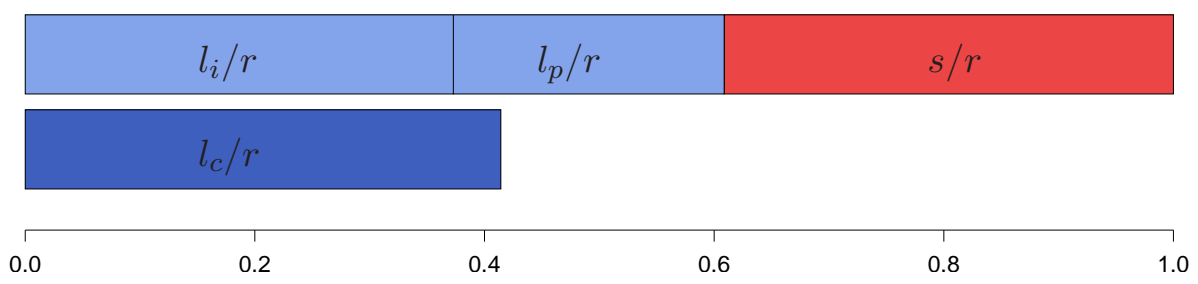

Figure 1: Average distribution of the runs into short runs and long runs and of the long runs into independent and prefixed long runs. Furthermore, the average ratio of long run changes is given.

consists of different corpora such as the true Canterbury Corpus, the Large Corpus and the Calgary Corpus. The corpora contain files of different types. In addition to text files such as books and papers there are source code files, pictures, office documents, object files, and many more. A description of the corpora can be found at [17].

In our tests we selected all the files from the Canterbury Corpus, the Large Corpus and the Calgary Corpus. We applied the Burrows-Wheeler transformation to each of these files. We chose a block size of $9 \cdot 10^{5}$ bytes, which is the default and also the maximum allowable block size in bzip2. If the file size exceeds the block size, then, as described above, the file is split into several blocks of the chosen size. This was the case for all the files of the Large Corpus. The sequences obtained from the Burrows-Wheeler transformation are the request sequences on which the list update algorithms have to be evaluated. Recall that each byte of the sequence forms a request. We remark that we actually do not compress files; instead we evaluate list update algorithms on these realistic benchmark sequences.

Table 3 in Appendix A shows the characteristics of our (transformed) request sequences. A graphical summary is depicted in Figure 1. The length of the request sequences differs vastly among the test instances. There are short sequences consisting of only 3721 requests (grammar. $1 \mathrm{sp}$ ) and long sequences of up to $9 \cdot 10^{5}$ references, which occur when a file is split into several blocks. The third column of Table 3 shows how many blocks were generated. Moreover, the number of different bytes (ASCII characters) requested differs vastly. In text files typically about 80 to 90 different characters are requested. In object files such as obj 1 and obj 2 of the Calgary Corpus all the 256 ASCII characters are referenced.

For each of the request sequences we have computed the values $r, s, l, l_{p}, l_{i}, l_{c}$ as introduced in Section 2. We have also computed values $f_{b}, f_{b}^{\prime}, f_{e}$ defined in Section 3.1 but, for brevity, do not show them. For better reading, instead of giving the absolute values of $r, s, l, l_{p}, l_{i}, l_{c}$ we show the interesting relations. Table 3 contains the exact values for each file while Figure 1 depicts the average values over all the files. First, it shows that among all the runs of a request sequence, about $60 \%$ to $65 \%$ are long runs. The fraction can go as high as $80 \%$ in the case of file trans in the Calgary Corpus. An exception is the file kennedy.$x 1 s$ in which only $10 \%-15 \%$ of the runs are long, indicating that the Burrows-Wheeler transformation does not work well on this file. Columns 7 and 8 of Table 3 and Figure 1 show the distribution of the prefixed and independent long runs among all the long runs. Again, in most cases, the majority of the long runs are independent long runs (fractions can go as high as $80 \%$ ), indicating that long runs are usually followed by long runs. Finally, the last column of Table 3 and Figure 1 show the number of long run changes relative to the number of long runs. Here the interesting observation is that the ratio $l_{c} / l$ is typically $5 \%$ to $10 \%$ higher than $l_{i} / l$. This demonstrates that the definition of $l_{c}$ was indeed sensible in Section 2 as it yields more expressive lower bounds on the cost of $O P T$, compared to $l_{i}$. A final remark is that, for files split into several blocks, all the numbers for the various blocks are consistent, i.e. the characteristics do not change within the file.

Performance results: We have executed the online algorithms analyzed in Section 3 on all the request sequences described above and recorded their cost. As for the randomized strategies BIT and COMB, they were executed 16 times on each sequence and, for any sequence, the average cost was taken. Since the 


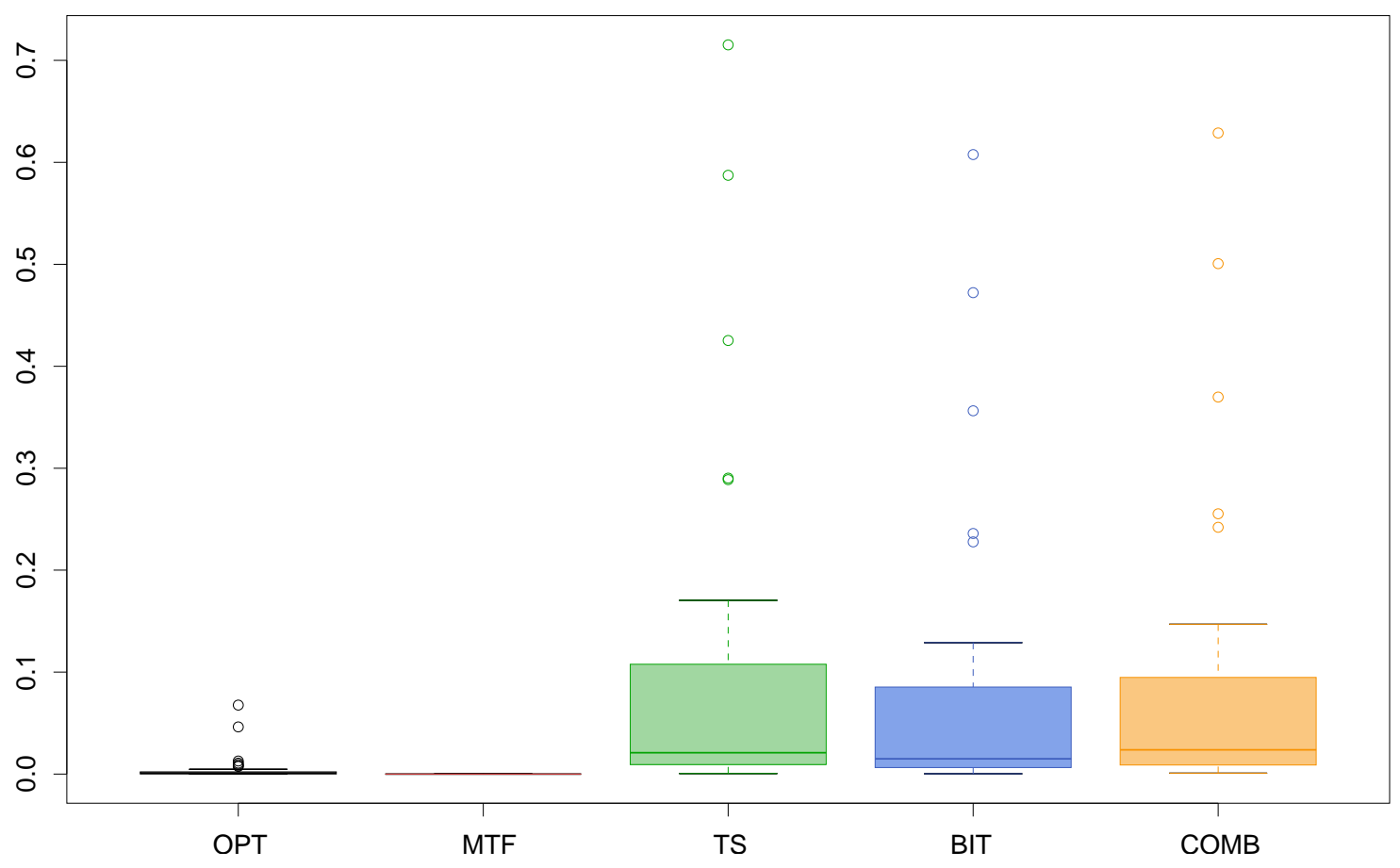

Figure 2: Relative errors of the upper bounds on the service costs given by Lemmas 1-5 when compared to the actual costs observed.

offline version of the list update problem is NP-hard [5] and the best known offline algorithm takes $O\left(2^{n} n ! m\right)$ time [34], where $n=|L|$ and $m=|\sigma|$, computing the true optimum offline cost is impossible for our request sequences. Therefore, we computed the pairwise optimum, which is equal to $\sum_{\{x, y\} \subseteq L, x \neq y} O P T\left(\sigma_{x y}\right)+|\sigma|$. This expression is usually used as approximation of the optimum offline cost, even in standard competitive analysis, see e.g. [1]. To evaluate the pairwise optimum, for each request sequence $\sigma$ and each pair $x, y$ of ASCII characters we derived the projected sequence $\sigma_{x y}$ and computed $O P T\left(\sigma_{x y}\right)$. The latter cost is easy to determine because an optimal offline algorithm for sequences on two-item lists is simple to state, cf. proof of Lemma 1. In the following figures and tables, OPT always refers to the pairwise optimum.

Table 4 in Appendix A and Figure 2 above represent the costs incurred by the algorithms on all the sequences. For each algorithm we compare the costs observed in the experiments to those implied by the theoretical bounds of Lemmas 1-5. For any $A \in\{M T F, T S, B I T, C O M B, O P T\}$, the columns headed $A^{*}$ in Table 4 record the experimentally observed cost. Using values $r, s, l, l_{i}, l_{p}, l_{c}, f_{b}, f_{b}^{\prime}, f_{e}$ for the various request sequences, Lemmas 1-5 yield theoretical bounds. Instead of reporting these bounds, we give the relative errors. The relative error, for a given strategy $A$ and request sequence $\sigma$, is the absolute value of the difference between the experimental and theoretical costs, divided by the experimental value. Figure 2 depicts the relative errors using box plots, which is a standard method to display numerical data. For each algorithm, the bold line within the box represents the median data point. The box includes $50 \%$ of the data points, where $25 \%$ is located above and $25 \%$ below the median. The upper (respectively lower) whisker is the maximum (respectively minimum) data point that can be found within a distance of 1.5 of the inter-quartile range. All other points are outliers. It shows that all the errors are very small, indicating that the bounds developed in Lemmas 1-5 very well approximate the experimentally observed cost. As for $O P T$, or the pairwise optimum, the average relative error is below $0.5 \%$. The incurred error for $M T F$ is 0 because the bound given in Lemma 2 is exact. For the other three online strategies, the average relative errors are between 


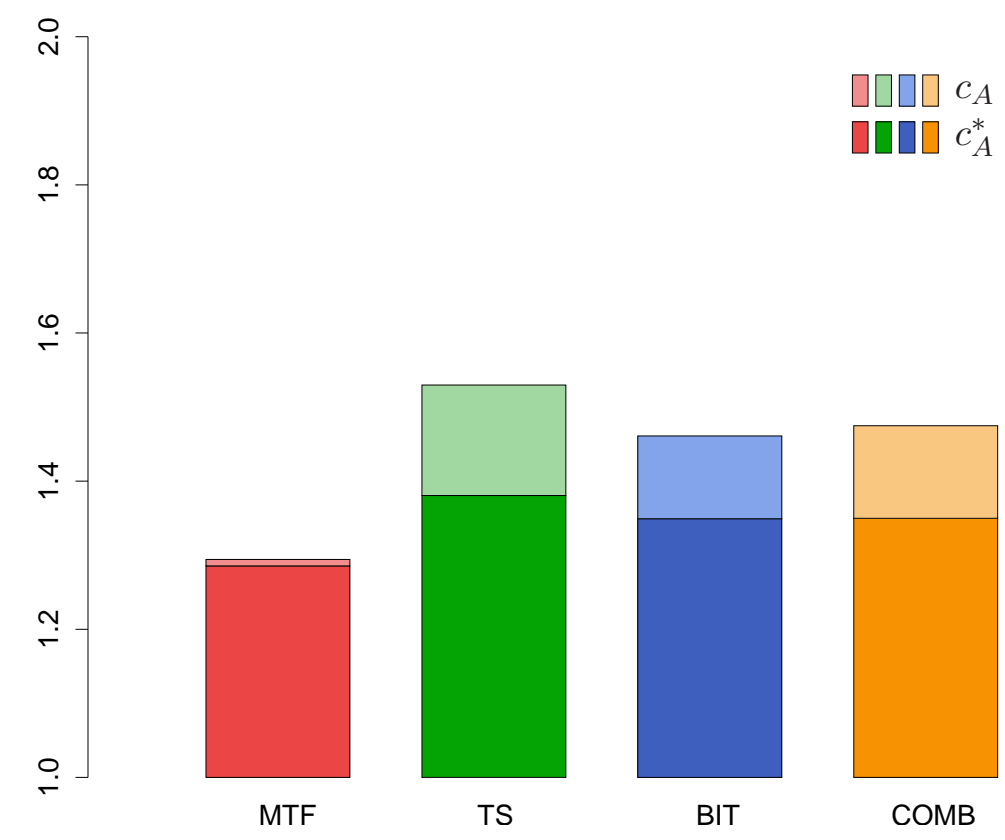

Figure 3: Average upper bounds $c_{A}$ on the performance ratios as implied by Theorems 1-4 compared to the average experimentally observed competitiveness $c_{A}^{*}$.

$7 \%$ and $8 \%$; the medians are even below $2 \%$. Observe that the errors cannot be 0 because Lemmas 3-5 give upper bounds on the service cost of the respective algorithms.

For comparison with previous experimental studies $[9,10]$ Table 5 shows the average service cost incurred by the algorithms on a single request. These average costs are all very small, typically in the range between 1.8 and 5, which confirms that the request sequences exhibit a high degree of locality and that the algorithms respond well to this property. In files such as kennedy.xIs, geo or obj1, which access the full ASCII character set, the access cost is a bit higher.

Table 6 in Appendix A as well as Figures 3 and 4 contain the main results of our experimental study. Table 6 presents, for each online algorithm and each of our request sequences, the experimentally observed competitiveness and compares it to the theoretical bounds developed in Theorems 1-4. For each algorithm $A \in\{M T F, T S, B I T, C O M B\}$, expression $c_{A}^{*}$ refers to the experimentally observed competitiveness on a given sequence, which is the actual cost incurred by $A$ divided by the cost of the pairwise optimum; the latter values are reported in Table 4. Expression $c_{A}$ refers to the theoretical bound. For each algorithm, the average performance values, over all files, are depicted in dark color (exp3rimental bounds) and light color (theoretical bounds) in Figure 3. Table 6 also shows the relative error between the experimental and theoretical expressions; a graphical representation using box plots is given in Figure 4. A first, very positive finding is that the experimentally observed and theoretical performance ratios are very close to each other. Hence our locality model and theoretical analyses are indeed sensible. The best results are achieved for MTF. Here the average relative error is below $0.7 \%$. For almost all of the files, the actual error is substantially smaller because four files in the Canterbury Corpus (fields.c, grammar.lsp, sum and xargs.1) contribute very higher errors to the average value. For the other three online algorithms the average relative errors are higher, ranging between $8 \%$ and $10 \%$, but these values are still reasonable. Again, for many files we have very small errors; high contributions in the average relative errors just come from the files fields.c, grammar. $1 \mathrm{sp}$ and xargs. 1. Figure 4 illustrates that the median error values are even below $3 \%$.

A second important result is that the experimentally observed competitiveness as well as the performance 


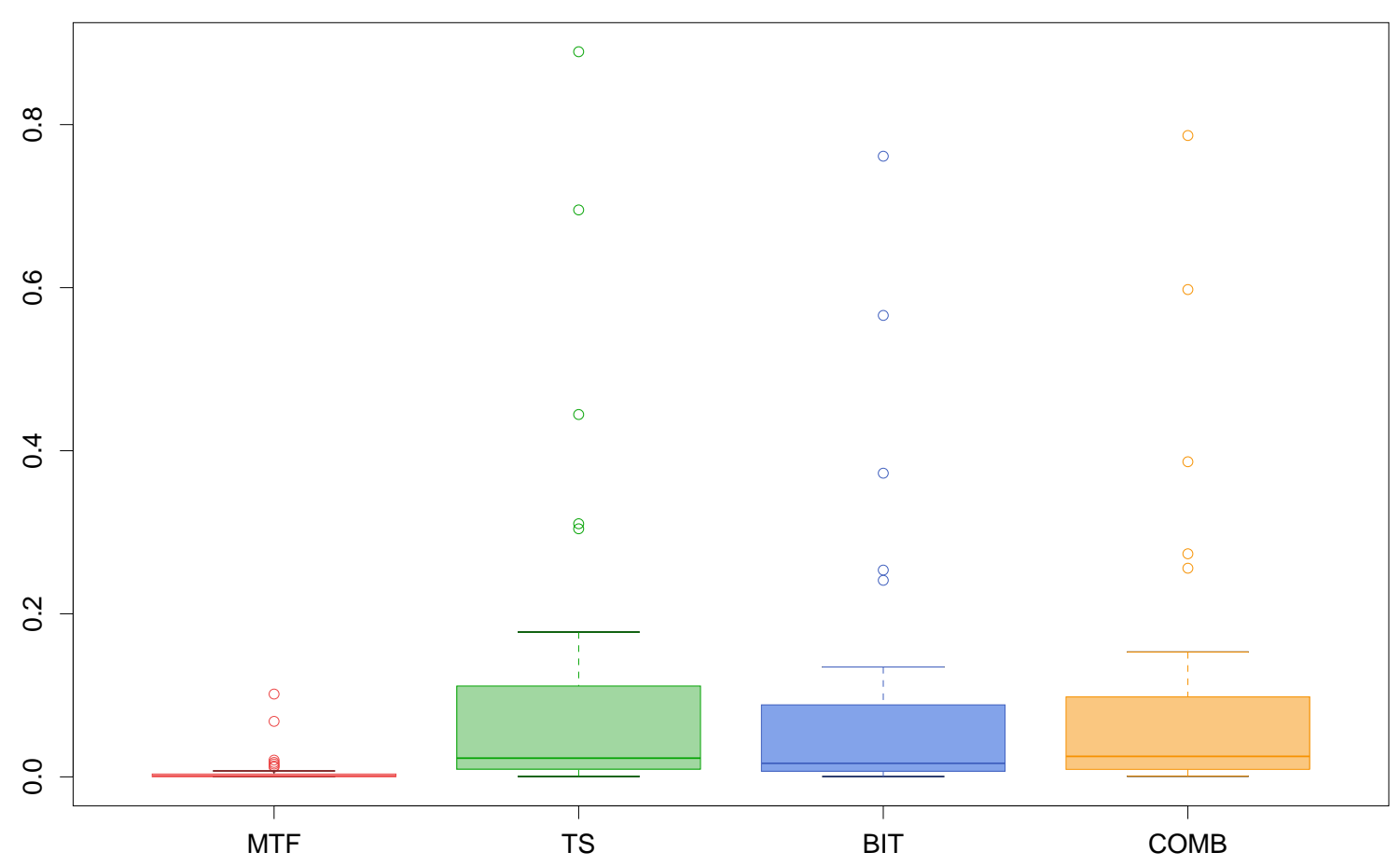

Figure 4: Relative errors of the upper bounds on the performance ratios as implied by Theorems $1-4$ when compared to the experimentally observed competitiveness.

ratios implied by Theorems 1-4 are much lower than the standard competitive ratios of the algorithms. Recall that $M T F$ and $T S$ are 2-competitive while $B I T$ and $C O M B$ achieve competitive ratios of 1.75 and 1.6, respectively. In our experiments, $M T F$ shows the best behavior with performance ratios between 1.2 and 1.3. An exception is the file kennedy.$x 1 s$ where the transformed request sequences do not exhibit a substantial degree of locality. The other three algorithms $T S, B I T$ and $C O M B$ are slightly worse, with ratios that are typically in the range between 1.3 and 1.6. There is no clear winner among these three algorithms. For each strategy there are some sequences where this strategy outperforms the other two. We note that for a few files such as fields.c, grammar. Isp or xargs. l, the theoretical bounds implied by Theorem 2-4 are higher than the standard competitive ratios of the algorithms. This is no contradiction. On these relatively short files, consisting of only a few thousands of requests, the initial, apparently unfortunate list ordering has a high influence of the overall service cost. The terms $f_{b}(\sigma)$ and $f_{b}^{\prime}(\sigma)$, which take into account such effects, are ignored in classical competitive analysis that evaluates the performance of algorithms on long sequences.

Table 7 in Appendix A and Figure 5 report on the performance of $M T F$ and BIT in terms of $\lambda$-locality. As argued in Section 3.3, TS and COMB achieve no improved competitiveness and hence are not listed. For algorithm $A \in\{M T F, B I T\}$, expression $c_{A}^{\lambda}$ refers to the theoretical bounds implied by Corollaries 1 and 2. We compare this value to the experimentally observed competitiveness $c_{A}^{*}$, reported in Table 6 , and compute relative errors. In order to get a reasonable set of numerical results, which can also be compared to that of Table 6, we treat each sequence as being a representative member of a class of inputs. (Of course, alternatively, one could also group sequences to form classes.) For both $M T F$ and BIT the competitive performance under $\lambda$-locality is higher than the theoretical bounds of Theorems 1 and 3 . This is not surprising because Corollaries 1 and 2 consider asymptotic algorithm performance, ignoring the request sequence length $|\sigma|$, i.e. an additive 1 per request, in both the online and offline cost. However, since the average service cost of the algorithms is small (cf. Table 5), the additive values of 1 make a difference in performance. For 


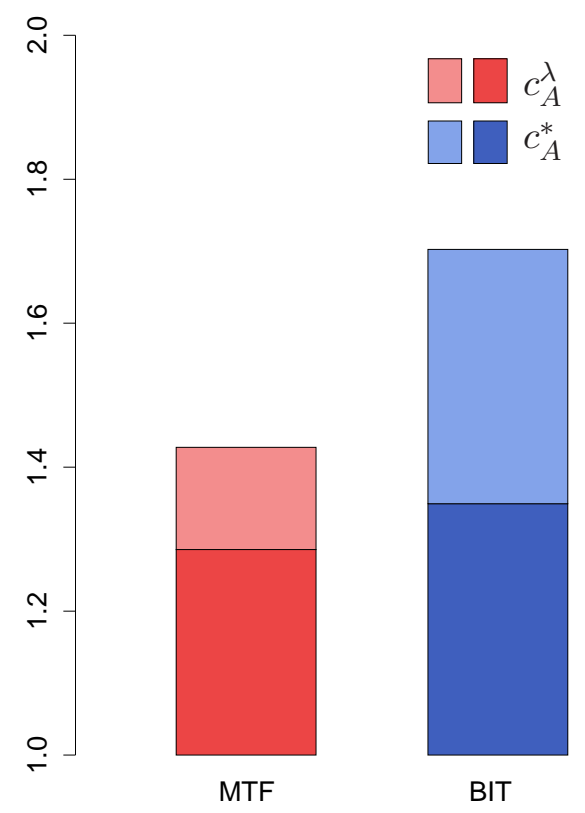

Figure 5: Average bounds on the performance ratios $c_{A}^{\lambda}$ for $A \in\{M T F, B I T\}$ as implied by Corollaries 1 and 2. For comparison, the corresponding average values of the experimentally observed ratios are represented by the darker colored bars.

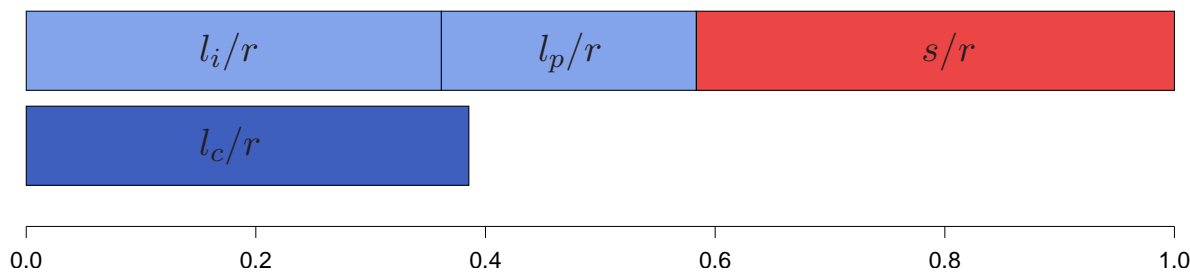

Figure 6: Average distribution of the runs of the memory access traces into short runs and long runs and of the long runs into independent and prefixed long runs. Furthermore, the average ratio of long run changes is given.

$M T F$ we have competitive values, in terms of $\lambda$-locality, that are typically between 1.2 and 1.5 , which is still considerably lower than the standard competitive ratio of 2. For BIT the values are usually above 1.6 and often reach even 1.75 .

\subsection{Memory accesses}

We now report on our experiments with memory accesses, thereby simulating dictionary operations. In these request sequences, each request is an access to a memory page. The alphabet is the set of different pages ever referenced in a given sequence. In contrast to the data compression experiments, where the list of 256 ASCII characters was fixed, here our self-organizing lists grow dynamically. Whenever a page is referenced for the first time, its address is inserted as new item into the list. This is simply done by appending the new item at the end of the list and executing the chosen list update strategy assuming a search request was issued.

Data sets and their locality characteristics: We selected traces that were generated by SPEC (Standard Performance Evaluation Corporation) which maintains widely-used benchmark collections for performance 


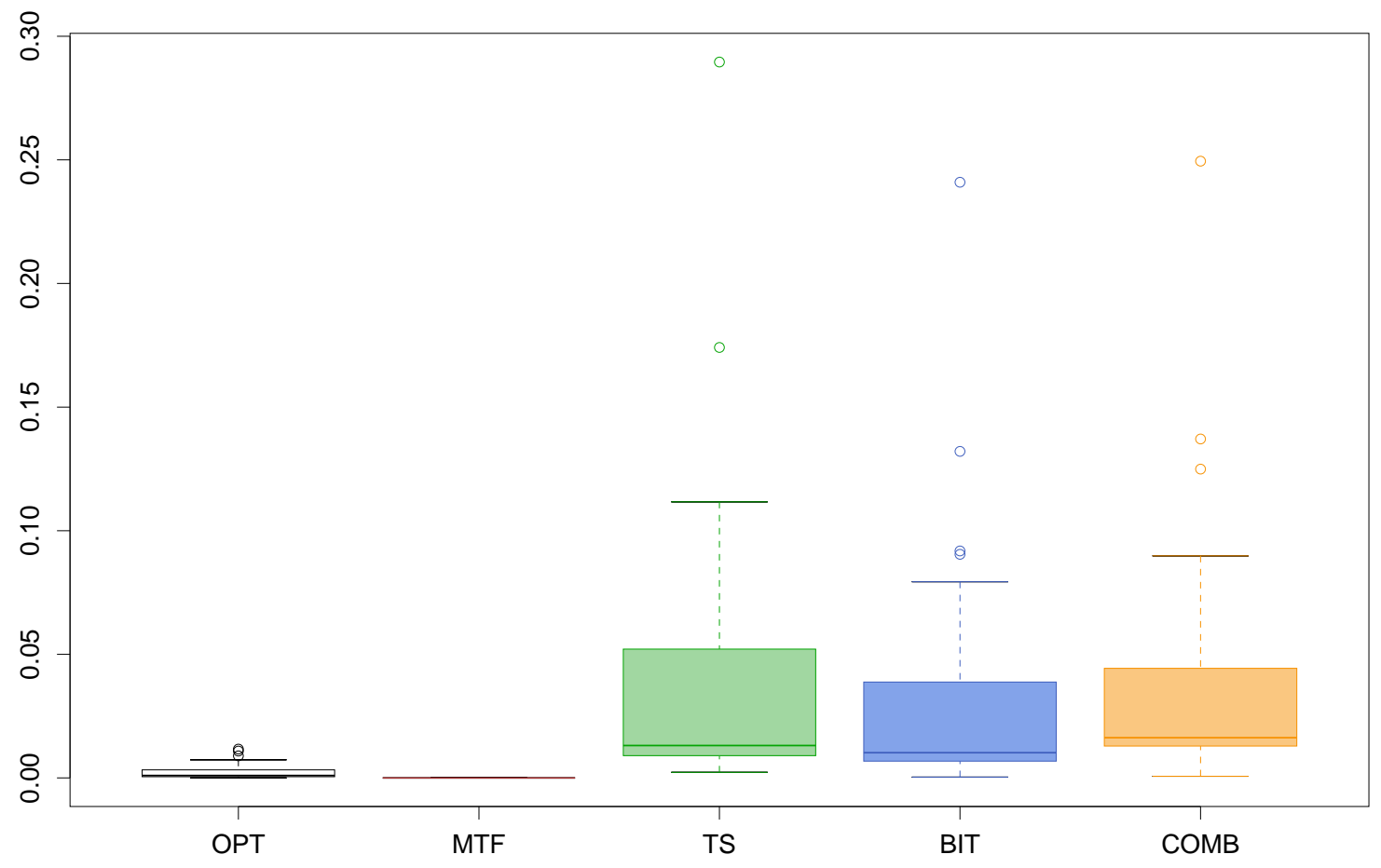

Figure 7: Relative errors of the upper bounds on the service costs on the memory access traces as implied by Lemmas 1-5.

evaluation [40]. The traces were generated by execution of the SPEC CPU2000 benchmark suite that contains a large variety of programs ranging from compilers, to word processors, to computer visualization programs, to name just a few. The programs were executed on a Pentium II processor running Redhat Linux 6.0. The resulting set of 47 memory access traces can be downloaded at the Bringham Young University Trace Distribution Center [15]. Instead of working with the whole traces which partly contain several hundreds of millions of requests, we considered the samples of the traces provided. These samples consist of the first section of the traces up to nearly 10.5 million requests. More information about the traces can be found at [40].

Table 8 in Appendix B and Figure 6 show the characteristics of the traces. The alphabet size differs vastly for the various traces and ranges from 251 (ammp) to 3151 (perl makerand). Interestingly, the locality characteristics are very similar to those of the data compression traces. This confirms the known fact that memory accesses do exhibit locality of reference. Among the runs of a sequence, typically about $60 \%$ are long runs. Recall that the numbers were about $10 \%$ to $15 \%$ higher in the case of data compression. In our test set only file mesa forms an exception with a fraction of $60 \%$ of short runs. Among the long runs, the majority forms independent long runs (fractions can be as high as $75 \%$ ). The ratio $l_{c} / l$ is typically $5 \%$ higher than $l_{i} / l$. We recall that in data compression this increase as well as the proportion of independent long runs was a bit higher. The reason is that the Burrows-Wheeler transformation generates sequences with a high degree of locality.

Performance results: As usual we have executed all our algorithms on each of the traces. The randomized algorithms were again executed 16 times on each input and the average cost was recorded. Table 9 in Appendix B and Figure 7 present the actual service costs of the algorithms and the relative errors incurred by the corresponding theoretical bounds of Lemmas 1-5. The average error of $O P T$, or the pairwise optimum, is less than $0.2 \%$. As for $M T F$, the error is 0 because our theoretical bound is exact. For the other three algorithms the average relative error is around 3\%, and hence even lower than in the case of the data compression 


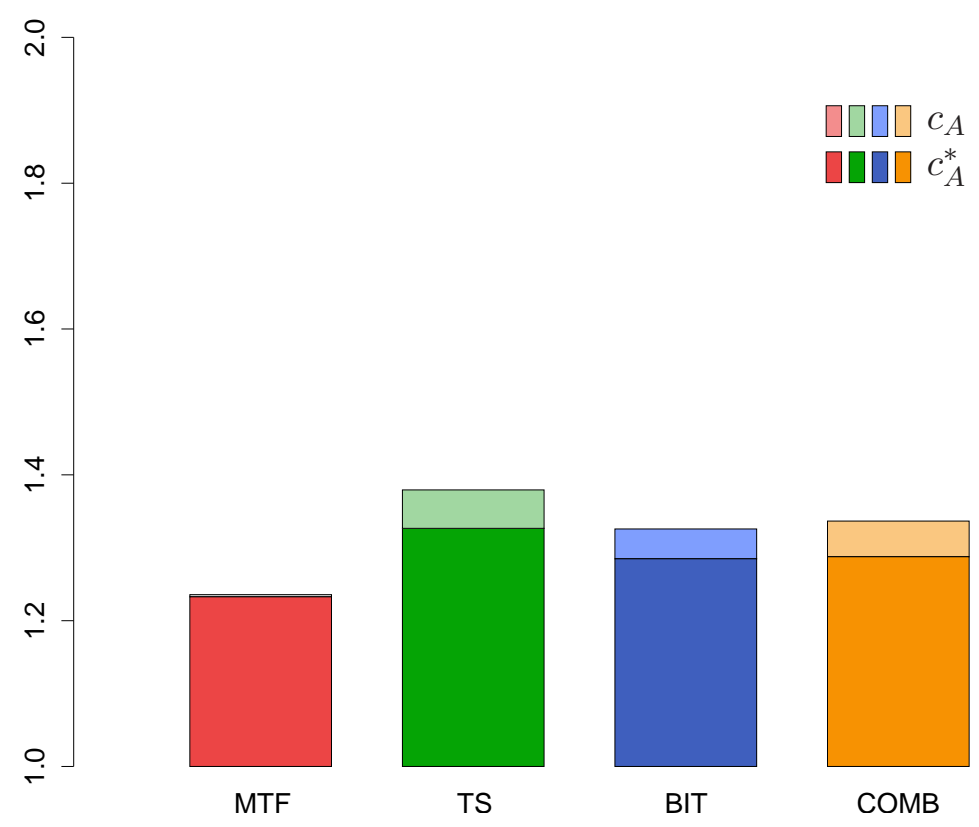

Figure 8: Average upper bounds $c_{A}$ on the performance ratios on the memory access traces, as implied by Theorems 1-4, compared to the average experimentally observed competitiveness $c_{A}^{*}$.

traces. The median error values are below $2 \%$, cf. Figure 7. Table 10 depicts the average service cost per request of the algorithms. We observe very small values between 2 and 3 uniformly over all the algorithms and traces. Again these values are even slightly lower than those of the data compression traces.

The most important results are presented in Table 11 in Appendix B as well as in Figures 8 and 9 below, which compare the experimentally observed competitiveness of the algorithms to the performance guarantees of Theorems 1-4. The relative errors are also computed. These errors are surprisingly small and again even lower than in the experiments with the data compression traces. For $M T F$ we observe an average relative error of $0.2 \%$. For the other algorithms the average relative errors are between $3 \%$ and $4 \%$; the media values are below $2 \%$. Furthermore, the performance ratios of the algorithms are very low. Algorithm MTF exhibits theoretical and experimental performance ratios that are typically in the range between 1.2 and 1.25. Hence these values are substantially smaller than $M T F$ 's competitiveness of 2. For the other three algorithms the performance ratios are a bit higher, typically ranging between 1.25 and 1.4. There are two exceptions, namely files mef and perl makerand where the ratios are higher. In general, BIT appears to be slightly better than $T S$ and $C O M B$ but the difference is marginal. For all the latter three algorithms, too, the experimentally observed and theoretical performance ratios are well below the competitive ratios of the strategies.

Finally Table 12 in Appendix B and Figure 10 below show the results for $\lambda$-locality. We assume again that each trace is a representative sample from a given application. The table shows the competitiveness of the algorithms, expressed in terms of $\lambda$, and the relative errors when the value is compared to the experimentally observed competitiveness shown in Table 12. As in the case of the experiments with data compression traces, $M T F$ exhibits performance guarantees that are well below the competitive ratio of 2; for BIT the guarantees are in the range 1.65-1.75 and hence not substantially below the standard competitiveness of 1.75 . We remark again that the performance ratios in Table 12 are higher than the bounds of Table 11 because Corollaries 1 and 2 ignore the request sequence length $|\sigma|$, i.e. an additive 1 per request, which makes a difference because the average cost of the algorithms is very low. Since the average service cost on the memory traces is even lower than on the data compression traces, the relative errors here are higher. 


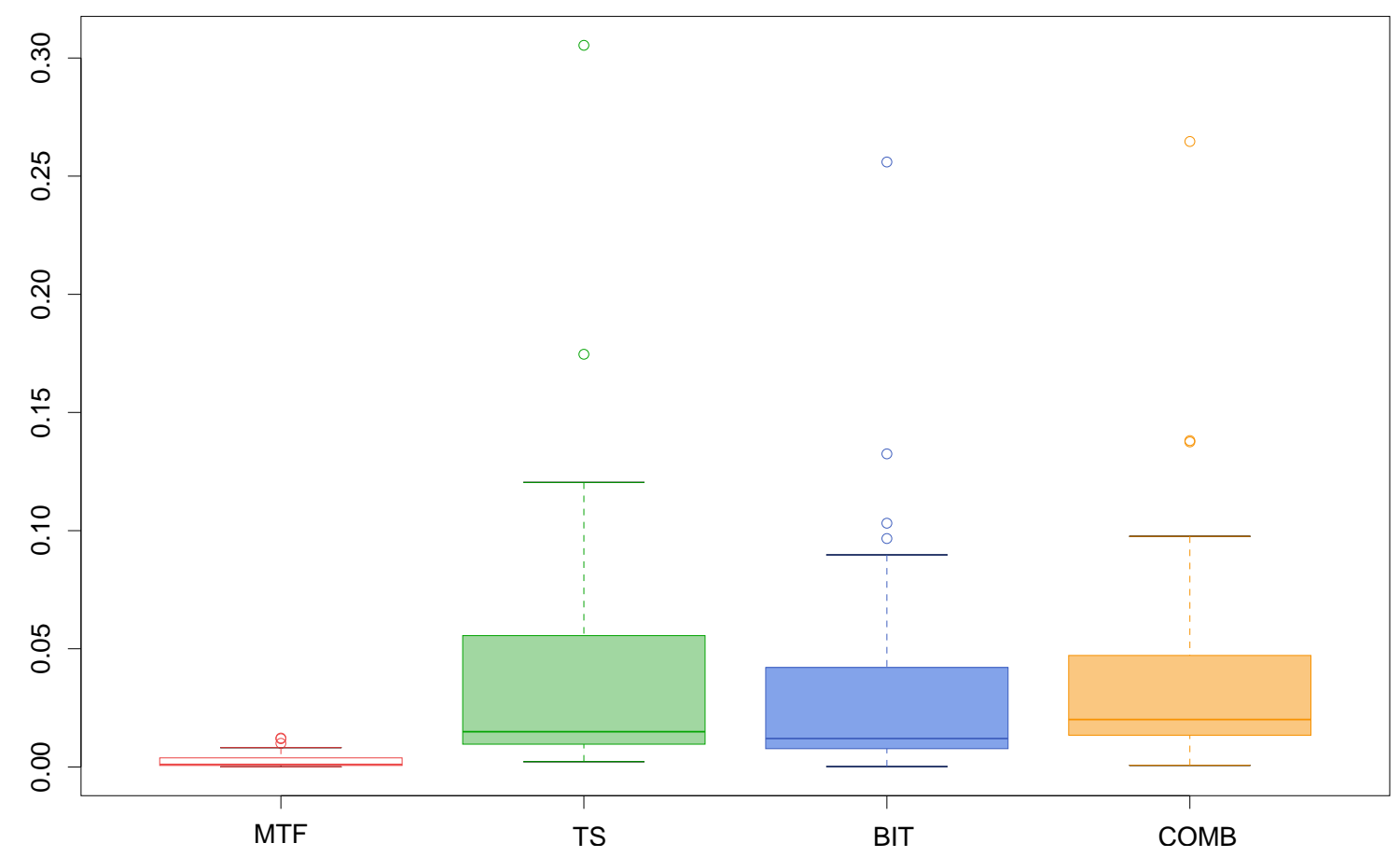

Figure 9: Relative errors of the upper bounds on the performance ratios on the memory access traces, as implied by Theorems $1-4$.

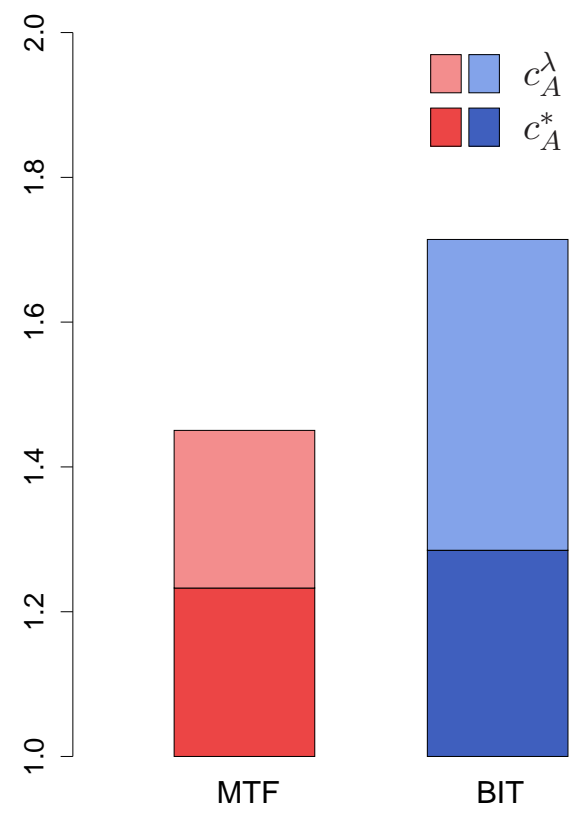

Figure 10: Average bounds on the performance ratios $c_{A}^{\lambda}$ for $A \in\{M T F, B I T\}$ for the memory access traces as implied by Corollaries 1 and 2. For comparison, the corresponding average values of the experimentally observed ratios are represented by the darker colored bars. 
Overall we observe the same qualitative results as in the tests with the data compression sequences. As mentioned before the results for the memory access traces are even more positive, i.e. the average relative errors between the theoretical an experimental bounds are smaller.

\section{Conclusions}

In this paper we have presented a new, powerful model of locality of reference that allows us analyze list update algorithms. We have developed refined theoretical performance guarantees for popular online algorithms. A main result is that Move-To-Front responds very well to locality of reference, which does not hold true for the other strategies examined. We have complemented the theoretical investigations by an extensive experimental study. It shows that the theoretically proven and experimentally observed performance guarantees match up to very small relative errors. We conjecture that, with respect to the competitive ratios, the gap between the theoretical and experimental bounds could be tightened even further if it were possible to derive better lower bounds on the optimum offline cost. More generally, an interesting working direction is to apply our locality model to other data structures problems. A natural candidate are splay trees [39]. A challenge is to analyze the cost of the search tree operations in terms of locality parameters.

\section{References}

[1] S. Albers. Improved randomized on-line algorithms for the list update problem. SIAM Journal on Computing, 27:670-681, 1998.

[2] S. Albers and M. Mitzenmacher. Average case analyses of list update algorithms, with applications to data compression. Algorithmica, 21:312-329, 1998.

[3] S. Albers, L.M. Favrholdt and O. Giel. On paging with locality of reference. Journal of Computer and System Sciences, 70:145-175, 2005.

[4] S. Albers, B. von Stengel and R. Werchner. A combined BIT and TIMESTAMP algorithm for the list update problem. Information Processing Letters, 56:135-139, 1995.

[5] C. Ambühl. Offline list update is NP-hard. Proc. 8th Annual European Symposium on Algorithms, Springer LNCS 1879, 42-51, 2000.

[6] C. Ambühl, B. Gärtner and B. von Stengel. A new lower bound for the list update problem in the partial cost model. Theoretical Computer Science, 268:3-16, 2001.

[7] S. Angelopoulos, R. Dorrigiv and A. López-Ortiz. On the separation and equivalence of paging strategies. 18th Annual ACM-SIAM Symposium on Discrete Algorithms, 229-237, 2007.

[8] S. Angelopoulos, R. Dorrigiv and A. López-Ortiz. List update with locality of reference: MTF outperforms all other algorithms. Proc. 8th Latin American Symposium on Theoretical Informatics, Springer LNCS 4957, 399-410, 2008.

[9] R. Bachrach and R. El-Yaniv. Online list accessing algorithms and their applications: Recent empirical evidence. Proc. 8th Annual ACM-SIAM Symposium on Discrete Algorithms, 53-62, 1997.

[10] R. Bachrach, R. El-Yaniv and M. Reinstädtler. On the competitive theory and practice of online list accessing algorithms. Algorithmica, 32:201-245, 2002.

[11] S. Ben-David, A. Borodin, R. Karp, G. Tardos, and A. Wigderson. On the power of randomization in on-line algorithms. Algorithmica, 11:2-14, 1994.

[12] J.L. Bentley and C.C. McGeoch. Amortized analyses of self-organizing sequential search heuristics. Communication of the ACM, 28:404-411, 1985.

[13] J.L. Bentley, D.S. Sleator, R.E. Tarjan and V.K. Wei. A locally adaptive data compression scheme. Communication of the ACM, 29:320-330, 1986. 
[14] A. Borodin, S. Irani, P. Raghavan and B. Schieber. Competitive paging with locality of reference. Journal of Computer and System Sciences, 50:244-258, 1995.

[15] BYU Trace Distribution Center, http://tds.cs.byu . edu/tds/index . jsp

[16] M. Burrows and D.J. Wheeler. A block-sorting lossless data compression algorithm. DEC SRC Research Report 124, 1994.

[17] The Canterbury Corpus, http: / / corpus.canterbury.ac.nz /

[18] F.R.K. Chung, D.J. Hajela and P.D. Seymour. Self-organizing sequential search and Hilbert's inequality. Proc. 17th Annual Symposium on the Theory of Computing, 217-223, 1985.

[19] R. Dorrigiv, M.R. Ehmsen and A. López-Ortiz. Parameterized analysis of paging and list update algorithms. Algorithmica, 71(2):330-353, 2015.

[20] R. Dorrigiv and A. López-Ortiz. On certain new models for paging with locality of reference. Proc. 2nd International Workshop on Algorithms and Computation (WALCOM), Springer LNCS 4921, 200-209, 2008.

[21] R. Dorrigiv and A. López-Ortiz. A new perspective on list update: Probabilistic locality and working set. Proc. 9th International Workshop on Approximation and Online Algorithms, Springer LNCS 7164, 150-163, 2011.

[22] R. Dorrigiv and A. López-Ortiz. List update with probabilistic locality of reference. Information Processing Letters, 112(13):540-543, 2012.

[23] R. Dorrigiv, A. López-Ortiz and J.I. Munro. List update algorithms for data compression. Proc. IEEE Data Compression Conference (DCC), 512, 2008.

[24] R. Dorrigiv, A. López-Ortiz, and J.I. Munro. An application of self-organizing data structures to compression. Proc. 8th International Symposium on Experimental Algorithms, Springer LNCS 5526, 137$148,2009$.

[25] A. Fiat and A. Karlin. Randomized and multipointer paging with locality of reference. Proc. 27th Annual ACM Symposium on Theory of Computing, 626-634, 1995.

[26] A. Fiat and M. Mendel. Truly online paging with locality of reference. Proc. 38rd Annual Symposium on Foundations of Computer Science, 326-335, 1997.

[27] G.H. Gonnet, J.I. Munro and H. Suwanda. Towards self-organizing linear search. Proc. 19th Annual IEEE Symposium on Foundations of Computer Science, 169-174, 1979.

[28] D. Grinberg, S. Rajagopalan, R. Venkatesan and V.K. Wei. Splay trees for data compression. Proc. 6th Annual ACM-SIAM Symposium on Discrete Algorithms, 522-530, 1995.

[29] J.H. Hester and D. S. Hirschberg. Self-organizing linear search. ACM Computing Surveys, 17:295-312. 1985.

[30] S. Irani. Two results on the list update problem. Information Processing Letters, 38:301-306, 1991.

[31] A. Karlin, S. Phillips und P. Raghavan. Markov paging. Proc. 33rd Annual Symposium on Foundations of Computer Science, 24-27, 1992.

[32] R. Karp and P. Raghavan. Personal communication cited in [35], 1990.

[33] E. Koutsoupias and C.H. Papadimitriou. Beyond competitive analysis. Proc. 35th Annual Symposium on Foundations of Computer Science 394-400, 1994.

[34] N. Reingold and J. Westbrook. Optimum off-line algorithms for the list update problem. Technical Report YALEU/DCS/TR-805, Yale University, 1990.

[35] N. Reingold, J. Westbrook and D.D. Sleator. Randomized competitive algorithms for the list update problem. Algorithmica, 11:15-32, 1994.

[36] R. Rivest. On self-organizing sequential search heuristics. Communication of the ACM, 19:63-67, 1976.

[37] J. Seward. bzip2 home page, http://www.bzip.org/

[38] D.D. Sleator and R.E. Tarjan. Amortized efficiency of list update and paging rules. Communications of the ACM, 28:202-208, 1985. 
[39] D.D. Sleator and R.E. Tarjan. Self-adjusting binary search trees. Journal of the ACM, 32:652-686, 1985.

[40] SPEC - Standard Performance Evaluation Corporation, http: / /www . spec .org/

[41] E. Torng. A unified analysis of paging and caching. Algorithmica, 20:175-200, 1998. 


\section{Appendix A}

\begin{tabular}{|c|c|c|c|c|c|c|c|c|c|}
\hline file & size & block & diff. bytes & $s / r$ & $l / r$ & $l_{p} / l$ & $l_{i} / l$ & $l_{c} / l$ & $\lambda$ \\
\hline \multicolumn{10}{|c|}{ The Canterbury Corpus } \\
\hline alice29.txt & 152089 & $\overline{1} 1$ & 74 & 0.3840 & 0.6160 & 0.4328 & 0.5672 & 0.6327 & 0.3898 \\
\hline asyoulik.txt & 125179 & 1 & 68 & 0.3959 & 0.6041 & 0.4447 & 0.5553 & 0.6267 & 0.3786 \\
\hline cp.html & 24603 & 1 & 86 & 0.3032 & 0.6968 & 0.3196 & 0.6804 & 0.7280 & 0.5072 \\
\hline fields.c & 11150 & 1 & 90 & 0.1801 & 0.8199 & 0.1546 & 0.8454 & 0.8671 & 0.7109 \\
\hline grammar.lsp & 3721 & 1 & 76 & 0.2287 & 0.7713 & 0.1328 & 0.8672 & 0.8819 & 0.6802 \\
\hline kennedy.xls & 1029744 & $\begin{array}{l}1 \\
2\end{array}$ & $\begin{array}{l}256 \\
236\end{array}$ & $\begin{array}{l}0.8817 \\
0.8500\end{array}$ & $\begin{array}{l}0.1183 \\
0.1500\end{array}$ & $\begin{array}{l}0.6483 \\
0.6026\end{array}$ & $\begin{array}{l}0.3517 \\
0.3974\end{array}$ & $\begin{array}{l}0.6612 \\
0.5977\end{array}$ & $\begin{array}{l}0.0782 \\
0.0896\end{array}$ \\
\hline lcet10.txt & 426754 & 1 & 84 & 0.3963 & 0.6037 & 0.4607 & 0.5393 & 0.6069 & 0.3664 \\
\hline plrabn12.txt & 481861 & 1 & 81 & 0.4336 & 0.5664 & 0.5108 & 0.4892 & 0.5695 & 0.3226 \\
\hline ptt5 & 513216 & 1 & 159 & 0.4434 & 0.5566 & 0.5505 & 0.4495 & 0.5237 & 0.2915 \\
\hline sum & 38240 & 1 & 255 & 0.3820 & 0.6180 & 0.3934 & 0.6066 & 0.6740 & 0.4165 \\
\hline xargs.1 & 4227 & 1 & 74 & 0.2503 & 0.7497 & 0.1801 & 0.8199 & 0.8440 & 0.6328 \\
\hline \multicolumn{10}{|c|}{ The Large Corpus } \\
\hline$\overline{\text { E.coli }}$ & 4638690 & $\begin{array}{l}1 \\
2 \\
3 \\
4 \\
5 \\
6\end{array}$ & $\begin{array}{l}4 \\
4 \\
4 \\
4 \\
4 \\
4\end{array}$ & $\begin{array}{l}0.4930 \\
0.4972 \\
0.4973 \\
0.4946 \\
0.4904 \\
0.4970\end{array}$ & $\begin{array}{l}0.5070 \\
0.5028 \\
0.5027 \\
0.5054 \\
0.5096 \\
0.5030\end{array}$ & $\begin{array}{l}0.5196 \\
0.5224 \\
0.5259 \\
0.5209 \\
0.5172 \\
0.5184\end{array}$ & $\begin{array}{l}0.4804 \\
0.4776 \\
0.4741 \\
0.4791 \\
0.4828 \\
0.4816\end{array}$ & $\begin{array}{l}0.6250 \\
0.6245 \\
0.6206 \\
0.6250 \\
0.6271 \\
0.6312\end{array}$ & $\begin{array}{l}0.3169 \\
0.3140 \\
0.3120 \\
0.3159 \\
0.3195 \\
0.3175\end{array}$ \\
\hline bible.txt & 4047392 & $\begin{array}{l}1 \\
2 \\
3 \\
4 \\
5\end{array}$ & $\begin{array}{l}62 \\
62 \\
63 \\
61 \\
63\end{array}$ & $\begin{array}{l}0.3971 \\
0.4002 \\
0.4217 \\
0.4046 \\
0.4142\end{array}$ & $\begin{array}{l}0.6029 \\
0.5998 \\
0.5783 \\
0.5954 \\
0.5858\end{array}$ & $\begin{array}{l}0.4608 \\
0.4665 \\
0.4968 \\
0.4707 \\
0.4797\end{array}$ & $\begin{array}{l}0.5392 \\
0.5335 \\
0.5032 \\
0.5293 \\
0.5203\end{array}$ & $\begin{array}{l}0.6102 \\
0.6054 \\
0.5815 \\
0.6027 \\
0.5965\end{array}$ & $\begin{array}{l}0.3679 \\
0.3631 \\
0.3363 \\
0.3589 \\
0.3494\end{array}$ \\
\hline world192.txt & 2473400 & $\begin{array}{l}1 \\
2 \\
3\end{array}$ & $\begin{array}{l}90 \\
84 \\
88\end{array}$ & $\begin{array}{l}0.3830 \\
0.3793 \\
0.3650\end{array}$ & $\begin{array}{l}0.6170 \\
0.6207 \\
0.6350\end{array}$ & $\begin{array}{l}0.4409 \\
0.4331 \\
0.4151\end{array}$ & $\begin{array}{l}0.5591 \\
0.5669 \\
0.5849\end{array}$ & $\begin{array}{l}0.6270 \\
0.6341 \\
0.6474\end{array}$ & $\begin{array}{l}0.3868 \\
0.3936 \\
0.4111\end{array}$ \\
\hline \multicolumn{10}{|c|}{ The Calgary Corpus } \\
\hline bib & 111261 & 1 & $\overline{881}$ & 0.3402 & 0.6598 & 0.3675 & 0.6325 & 0.6891 & 0.4547 \\
\hline book1 & 768771 & 1 & 82 & 0.4391 & 0.5609 & 0.5271 & 0.4729 & 0.5533 & 0.3104 \\
\hline book2 & 610856 & 1 & 96 & 0.3835 & 0.6165 & 0.4395 & 0.5605 & 0.6250 & 0.3853 \\
\hline geo & 102400 & 1 & 256 & 0.4829 & 0.5171 & 0.5446 & 0.4554 & 0.5741 & 0.2969 \\
\hline news & 377109 & 1 & 98 & 0.3498 & 0.6502 & 0.3964 & 0.6036 & 0.6585 & 0.4282 \\
\hline obj1 & 21504 & 1 & 256 & 0.4049 & 0.5951 & 0.4339 & 0.5661 & 0.6453 & 0.3840 \\
\hline obj2 & 246814 & 1 & 256 & 0.3433 & 0.6567 & 0.3852 & 0.6148 & 0.6736 & 0.4424 \\
\hline paper1 & 53161 & 1 & 95 & 0.2966 & 0.7034 & 0.3035 & 0.6965 & 0.7405 & 0.5208 \\
\hline paper2 & 82199 & 1 & 91 & 0.3535 & 0.6465 & 0.3849 & 0.6151 & 0.6729 & 0.4351 \\
\hline paper3 & 46526 & 1 & 84 & 0.3323 & 0.6677 & 0.3504 & 0.6496 & 0.7042 & 0.4702 \\
\hline paper4 & 13286 & 1 & 80 & 0.2773 & 0.7227 & 0.2674 & 0.7326 & 0.7728 & 0.5585 \\
\hline paper5 & 11954 & 1 & 91 & 0.2690 & 0.7310 & 0.2569 & 0.7431 & 0.7811 & 0.5710 \\
\hline paper6 & 38105 & 1 & 93 & 0.2757 & 0.7243 & 0.2786 & 0.7214 & 0.7611 & 0.5512 \\
\hline pic & 513216 & 1 & 159 & 0.4434 & 0.5566 & 0.5505 & 0.4495 & 0.5237 & 0.2915 \\
\hline progc & 39611 & 1 & 92 & 0.2876 & 0.7124 & 0.3004 & 0.6996 & 0.7427 & 0.5291 \\
\hline progl & 71646 & 1 & 87 & 0.2652 & 0.7348 & 0.2742 & 0.7258 & 0.7613 & 0.5594 \\
\hline progp & 49379 & 1 & 89 & 0.2135 & 0.7865 & 0.2086 & 0.7914 & 0.8179 & 0.6433 \\
\hline trans & 93695 & 1 & 99 & 0.1964 & 0.8036 & 0.1949 & 0.8051 & 0.8278 & 0.6652 \\
\hline
\end{tabular}

Table 3: Characteristics of the files and the transformed request sequences. File size (in bytes), partitioning in blocks, number of different bytes per block and statistics on the runs. 


\begin{tabular}{|c|c|c|c|c|c|c|c|c|c|c|}
\hline file & $O P T^{*}$ & $f_{O P T}$ & $M T F^{*}$ & $f_{M T F}$ & $T S^{*}$ & $f_{T S}$ & $B I T^{*}$ & $f_{B I T}$ & $C O M B^{*}$ & $f_{C O M B}$ \\
\hline \multicolumn{11}{|c|}{ The Canterbury Corpus } \\
\hline -alice29.txt & 392895 & 0.0013 & 502868 & $\overline{00}$ & 522306 & 0.0431 & 526456.9375 & 0.0319 & 524126.3125 & $\overline{0.0371}$ \\
\hline asyoulik.txt & 362574 & 0.0002 & 474180 & 0 & 487663 & 0.0436 & 494001.6875 & 0.0317 & 492105.3125 & 0.0353 \\
\hline cp.html & 104158 & 0.0031 & 133635 & 0 & 151906 & 0.1704 & 147630.9375 & 0.1289 & 147249.4375 & 0.1469 \\
\hline fields.c & 42524 & 0.0075 & 49582 & 0 & 65868 & 0.4253 & 58897.1250 & 0.3563 & 60363.6875 & 0.3698 \\
\hline grammar.lsp & 19391 & 0.0676 & 22572 & 0 & 30722 & 0.7153 & 27150.8125 & 0.6077 & 27910.2500 & 0.6287 \\
\hline kennedy.xls & $\begin{array}{r}12472268 \\
939959\end{array}$ & $\begin{array}{l}0.0010 \\
0.0047\end{array}$ & $\begin{array}{r}22354897 \\
1621774\end{array}$ & $\begin{array}{l}0 \\
0\end{array}$ & $\begin{array}{r}21597020 \\
1558178\end{array}$ & $\begin{array}{l}0.0012 \\
0.0250\end{array}$ & $\begin{array}{r}18080322.1875 \\
1340384.5000\end{array}$ & $\begin{array}{l}0.0004 \\
0.0202\end{array}$ & $\begin{array}{r}18274572.2500 \\
1356081.5625\end{array}$ & $\begin{array}{l}0.0285 \\
0.0423\end{array}$ \\
\hline lcet10.txt & 1027207 & 0.0003 & 1311293 & 0 & 1341239 & 0.0196 & 1361943.1875 & 0.0147 & 1355707.1250 & 0.0172 \\
\hline plrabn12.txt & 1282558 & 0.0003 & 1698414 & 0 & 1671403 & 0.0150 & 1729810.9375 & 0.0108 & 1715711.5000 & 0.0130 \\
\hline $\mathrm{ptt5}$ & 942890 & 0.0019 & 1180995 & 0 & 1131896 & 0.0224 & 1186887.9375 & 0.0155 & 1177437.5625 & 0.0155 \\
\hline sum & 337864 & 0.0128 & 460760 & 0 & 505699 & 0.0771 & 498365.5625 & 0.0533 & 496601.2500 & 0.0650 \\
\hline xargs. 1 & 23181 & 0.0463 & 28233 & 0 & 35967 & 0.5873 & 33074.9375 & 0.4722 & 33568.1875 & 0.5006 \\
\hline \multicolumn{11}{|c|}{ The Large Corpus } \\
\hline E.coli & $\begin{array}{r}1711786 \\
1727309 \\
1717674 \\
1719056 \\
1702239 \\
267290\end{array}$ & $\begin{array}{l}<10^{-4} \\
<10^{-4} \\
<10^{-4} \\
<10^{-4} \\
<10^{-4} \\
<10^{-4}\end{array}$ & $\begin{array}{r}2133228 \\
2159514 \\
2146793 \\
2145202 \\
2116237 \\
334220\end{array}$ & $\begin{array}{l}0 \\
0 \\
0 \\
0 \\
0 \\
0\end{array}$ & $\begin{array}{r}2108144 \\
2130515 \\
2113718 \\
2118336 \\
2094276 \\
329993\end{array}$ & $\begin{array}{l}0.0006 \\
0.0006 \\
0.0006 \\
0.0006 \\
0.0006 \\
0.0037\end{array}$ & $\begin{array}{r}2131150.5625 \\
2153816.5625 \\
2139920.1250 \\
2141468.1250 \\
2116540.4375 \\
333196.8750\end{array}$ & $\begin{array}{l}0.0004 \\
0.0004 \\
0.0005 \\
0.0005 \\
0.0004 \\
0.0030\end{array}$ & $\begin{array}{r}2125126.1250 \\
2147982.0000 \\
2133448.0625 \\
2135649.0000 \\
2111176.0625 \\
332557.5625\end{array}$ & $\begin{array}{l}0.0011 \\
0.0010 \\
0.0011 \\
0.0011 \\
0.0008 \\
0.0032\end{array}$ \\
\hline bible.txt & $\begin{array}{r}1690008 \\
1756850 \\
1837709 \\
1793291 \\
975056\end{array}$ & $\begin{array}{r}<10^{-4} \\
<10^{-4} \\
<10^{-4} \\
<10^{-4} \\
\\
\quad 0.0001\end{array}$ & $\begin{array}{l}2059346 \\
2161595 \\
2308138 \\
2219158 \\
1233963\end{array}$ & $\begin{array}{l}0 \\
0 \\
0 \\
0 \\
0\end{array}$ & $\begin{array}{l}2105108 \\
2203033 \\
2303754 \\
2256058 \\
1243358\end{array}$ & $\begin{array}{l}0.0091 \\
0.0087 \\
0.0085 \\
0.0084 \\
0.0157\end{array}$ & $\begin{array}{l}2129224.1250 \\
2233457.5625 \\
2360623.0625 \\
2289041.1250 \\
1268445.0000\end{array}$ & $\begin{array}{l}0.0065 \\
0.0062 \\
0.0060 \\
0.0064 \\
0.0113\end{array}$ & $\begin{array}{l}2121837.0625 \\
2224340.5000 \\
2346456.5000 \\
2280334.7500 \\
1261998.3750\end{array}$ & $\begin{array}{l}0.0082 \\
0.0081 \\
0.0077 \\
0.0077 \\
0.0133\end{array}$ \\
\hline world192.txt & $\begin{array}{l}2013201 \\
2031383 \\
1558549 \\
\end{array}$ & $\begin{array}{l}0.0002 \\
0.0001 \\
0.0002 \\
\end{array}$ & $\begin{array}{l}2510591 \\
2528823 \\
1933008 \\
\end{array}$ & $\begin{array}{l}0 \\
0 \\
0\end{array}$ & $\begin{array}{l}2616268 \\
2652556 \\
2057427 \\
\end{array}$ & $\begin{array}{l}0.0103 \\
0.0096 \\
0.0129 \\
\end{array}$ & $\begin{array}{l}2629476.6875 \\
2655963.0000 \\
2046894.1875\end{array}$ & $\begin{array}{l}0.0075 \\
0.0072 \\
0.0098 \\
\end{array}$ & $\begin{array}{l}2617647.7500 \\
2645196.6875 \\
2040235.3125 \\
\end{array}$ & $\begin{array}{l}0.0116 \\
0.0115 \\
0.0148 \\
\end{array}$ \\
\hline \multicolumn{11}{|c|}{ The Calgary Corpus } \\
\hline bib & 310092 & 0.0007 & 388932 & 0 & 427473 & 0.0587 & 418736.6250 & 0.0451 & 418474.8125 & 0.0529 \\
\hline book1 & 1998680 & 0.0003 & 2651634 & 0 & 2582324 & 0.0096 & 2689370.6875 & 0.0069 & 2662206.8750 & 0.0096 \\
\hline book2 & 1559892 & 0.0002 & 1986645 & 0 & 2076699 & 0.0138 & 2086792.3750 & 0.0103 & 2075630.1875 & 0.0155 \\
\hline geo & 2499234 & 0.0020 & 3797973 & 0 & 3615473 & 0.0098 & 3785313.6250 & 0.0062 & 3780945.8125 & 0.0010 \\
\hline news & 1396286 & 0.0002 & 1809619 & 0 & 1990178 & 0.0150 & 1960421.5625 & 0.0112 & 1950057.9375 & 0.0204 \\
\hline obj1 & 372864 & 0.0093 & 529522 & 0 & 554319 & 0.0739 & 560958.7500 & 0.0488 & 556354.1250 & 0.0600 \\
\hline obj2 & 2085629 & 0.0015 & 2796806 & 0 & 3172590 & 0.0106 & 3089426.6250 & 0.0085 & 3050721.6250 & 0.0272 \\
\hline paper1 & 182349 & 0.0019 & 226971 & 0 & 265066 & 0.1097 & 252272.8750 & 0.0867 & 252736.3125 & 0.1006 \\
\hline paper2 & 244978 & 0.0027 & 313512 & 0 & 337465 & 0.0813 & 334597.8750 & 0.0614 & 334155.4375 & 0.0687 \\
\hline paper3 & 157773 & 0.0024 & 201921 & 0 & 223426 & 0.1148 & 218574.8125 & 0.0877 & 218969.8750 & 0.0961 \\
\hline paper4 & 57123 & 0.0080 & 72257 & 0 & 84729 & 0.2888 & 80642.8750 & 0.2278 & 81351.9375 & 0.2421 \\
\hline paper5 & 61211 & 0.0107 & 77312 & 0 & 92995 & 0.2903 & 87205.7500 & 0.2359 & 87804.1875 & 0.2553 \\
\hline paper6 & 138675 & 0.0018 & 171298 & 0 & 204572 & 0.1417 & 192904.6250 & 0.1122 & 193633.4375 & 0.1277 \\
\hline pic & 942890 & 0.0019 & 1180995 & 0 & 1131896 & 0.0224 & 1186887.9375 & 0.0155 & 1177437.5625 & 0.0155 \\
\hline progc & 154882 & 0.0017 & 194241 & 0 & 228571 & 0.1238 & 217648.1250 & 0.0966 & 217821.8125 & 0.1124 \\
\hline progl & 180236 & 0.0014 & 213958 & 0 & 252752 & 0.1057 & 238902.5625 & 0.0840 & 240581.9375 & 0.0935 \\
\hline progp & 135749 & 0.0017 & 156964 & 0 & 199299 & 0.1364 & 181676.0000 & 0.1125 & 183934.0000 & 0.1253 \\
\hline trans & 245167 & 0.0018 & 278104 & 0 & 361727 & 0.0850 & 325851.5625 & 0.0709 & 331187.3750 & 0.0799 \\
\hline
\end{tabular}

Table 4: Actual service costs incurred by the algorithms. Comparison of the actual costs to the bounds of Lemmas $1-5$ yields the relative errors $f_{A}$, for the various strategies $A$. 


\begin{tabular}{|c|c|c|c|c|c|}
\hline file & $\overline{O P T^{*}}$ & $\overline{M T F^{*}}$ & $\overline{T S^{*}}$ & $\overline{B I T^{*}}$ & $\overline{C O M B^{*}}$ \\
\hline \multicolumn{6}{|c|}{ The Canterbury Corpus } \\
\hline alice29.txt & 2.5833 & 3.3064 & 3.4342 & 3.4615 & 3.4462 \\
\hline asyoulik.txt & 2.8964 & 3.7880 & 3.8957 & 3.9464 & 3.9312 \\
\hline cp.html & 4.2335 & 5.4317 & 6.1743 & 6.0005 & 5.9850 \\
\hline fields.c & 3.8138 & 4.4468 & 5.9074 & 5.2823 & 5.4138 \\
\hline grammar.lsp & 5.2112 & 6.0661 & 8.2564 & 7.2966 & 7.5007 \\
\hline kennedy.xls & 13.8581 & 24.8388 & 23.9967 & 20.0892 & 20.3051 \\
\hline & 7.2447 & 12.4998 & 12.0096 & 10.3310 & 10.4520 \\
\hline lcet10.txt & 2.4070 & 3.0727 & 3.1429 & 3.1914 & 3.1768 \\
\hline plrabn12.txt & 2.6617 & 3.5247 & 3.4686 & 3.5899 & 3.5606 \\
\hline $\mathrm{ptt5}$ & 1.8372 & 2.3012 & 2.2055 & 2.3126 & 2.2942 \\
\hline sum & 8.8354 & 12.0492 & 13.2243 & 13.0326 & 12.9864 \\
\hline xargs. 1 & 5.4840 & 6.6792 & 8.5089 & 7.8247 & 7.9414 \\
\hline \multicolumn{6}{|c|}{ The Large Corpus } \\
\hline \multirow[t]{6}{*}{ E.coli } & 1.9020 & 2.3703 & 2.3424 & 2.3679 & 2.3613 \\
\hline & 1.9192 & 2.3995 & 2.3672 & 2.3931 & 2.3866 \\
\hline & 1.9085 & 2.3853 & 2.3486 & 2.3777 & 2.3705 \\
\hline & 1.9101 & 2.3836 & 2.3537 & 2.3794 & 2.3729 \\
\hline & 1.8914 & 2.3514 & 2.3270 & 2.3517 & 2.3458 \\
\hline & 1.9272 & 2.4098 & 2.3794 & 2.4025 & 2.3978 \\
\hline \multirow[t]{5}{*}{ bible.txt } & 1.8778 & 2.2882 & 2.3390 & 2.3658 & 2.3576 \\
\hline & 1.9521 & 2.4018 & 2.4478 & 2.4816 & 2.4715 \\
\hline & 2.0419 & 2.5646 & 2.5597 & 2.6229 & 2.6072 \\
\hline & 1.9925 & 2.4657 & 2.5067 & 2.5434 & 2.5337 \\
\hline & 2.1794 & 2.7581 & 2.7791 & 2.8352 & 2.8208 \\
\hline \multirow[t]{3}{*}{ world192.txt } & 2.2369 & 2.7895 & 2.9070 & 2.9216 & 2.9085 \\
\hline & 2.2571 & 2.8098 & 2.9473 & 2.9511 & 2.9391 \\
\hline & 2.3144 & 2.8705 & 3.0553 & 3.0396 & 3.0298 \\
\hline \multicolumn{6}{|c|}{ The Calgary Corpus } \\
\hline bib & 2.7871 & 3.4957 & 3.8421 & 3.7636 & 3.7612 \\
\hline book1 & 2.5998 & 3.4492 & 3.3590 & 3.4983 & 3.4629 \\
\hline book2 & 2.5536 & 3.2522 & 3.3997 & 3.4162 & 3.3979 \\
\hline geo & 24.4066 & 37.0896 & 35.3074 & 36.9660 & 36.9233 \\
\hline news & 3.7026 & 4.7987 & 5.2775 & 5.1986 & 5.1711 \\
\hline obj1 & 17.3393 & 24.6243 & 25.7775 & 26.0863 & 25.8721 \\
\hline obj2 & 8.4502 & 11.3316 & 12.8542 & 12.5172 & 12.3604 \\
\hline paper1 & 3.4301 & 4.2695 & 4.9861 & 4.7455 & 4.7542 \\
\hline paper2 & 2.9803 & 3.8141 & 4.1055 & 4.0706 & 4.0652 \\
\hline paper3 & 3.3911 & 4.3400 & 4.8022 & 4.6979 & 4.7064 \\
\hline paper4 & 4.2995 & 5.4386 & 6.3773 & 6.0698 & 6.1231 \\
\hline paper5 & 5.1205 & 6.4675 & 7.7794 & 7.2951 & 7.3452 \\
\hline paper6 & 3.6393 & 4.4954 & 5.3686 & 5.0624 & 5.0816 \\
\hline pic & 1.8372 & 2.3012 & 2.2055 & 2.3126 & 2.2942 \\
\hline progc & 3.9101 & 4.9037 & 5.7704 & 5.4946 & 5.4990 \\
\hline progl & 2.5156 & 2.9863 & 3.5278 & 3.3345 & 3.3579 \\
\hline progp & 2.7491 & 3.1788 & 4.0361 & 3.6792 & 3.7249 \\
\hline trans & 2.6166 & 2.9682 & 3.8607 & 3.4778 & 3.5347 \\
\hline
\end{tabular}

Table 5: Average service cost per request incurred by the algorithms. 


\begin{tabular}{|c|c|c|c|c|c|c|c|c|c|c|c|c|}
\hline file & $c_{M T F}$ & $c_{M T F}^{*}$ & $f_{c_{M T F}}$ & $c_{T S}$ & $c_{T S}^{*}$ & $f_{c_{T S}}$ & $c_{B I T}$ & $c_{B I T}^{*}$ & $f_{c_{B I T}}$ & $c_{C O M B}$ & $c_{C O M B}^{*}$ & $f_{C_{C O M B}}$ \\
\hline \multicolumn{13}{|c|}{ The Canterbury Corpus } \\
\hline alice29.txt & 1.2820 & 1.2799 & 0.0016 & 1.3890 & 1.3294 & 0.0449 & 1.3847 & 1.3399 & 0.0334 & 1.3856 & 1.3340 & 0.0387 \\
\hline asyoulik.txt & 1.3084 & 1.3078 & 0.0004 & 1.4043 & 1.3450 & 0.0441 & 1.4061 & 1.3625 & 0.0320 & 1.4058 & 1.3573 & 0.0357 \\
\hline cp.html & 1.2908 & 1.2830 & 0.0061 & 1.7174 & 1.4584 & 0.1776 & 1.6084 & 1.4174 & 0.1347 & 1.6302 & 1.4137 & 0.1531 \\
\hline fields.c & 1.1816 & 1.1660 & 0.0134 & 2.2373 & 1.5490 & 0.4444 & 1.9008 & 1.3850 & 0.3724 & 1.9681 & 1.4195 & 0.3864 \\
\hline grammar.lsp & 1.2822 & 1.1640 & 0.1015 & 2.9935 & 1.5843 & 0.8894 & 2.4660 & 1.4002 & 0.7612 & 2.5715 & 1.4393 & 0.7866 \\
\hline kennedy.xls & 1.7944 & 1.7924 & 0.0011 & 1.7357 & 1.7316 & 0.0023 & 1.4518 & 1.4496 & 0.0015 & 1.5086 & 1.4652 & 0.0296 \\
\hline & 1.7380 & 1.7254 & 0.0073 & 1.7116 & 1.6577 & 0.0325 & 1.4642 & 1.4260 & 0.0268 & 1.5137 & 1.4427 & 0.0492 \\
\hline lcet10.txt & 1.2770 & 1.2766 & 0.0004 & 1.3317 & 1.3057 & 0.0199 & 1.3458 & 1.3259 & 0.0150 & 1.3430 & 1.3198 & 0.0176 \\
\hline plrabn12.txt & 1.3248 & 1.3242 & 0.0004 & 1.3233 & 1.3032 & 0.0154 & 1.3638 & 1.3487 & 0.0112 & 1.3557 & 1.3377 & 0.0134 \\
\hline $\mathrm{ptt} 5$ & 1.2570 & 1.2525 & 0.0036 & 1.2317 & 1.2005 & 0.0261 & 1.2821 & 1.2588 & 0.0185 & 1.2720 & 1.2488 & 0.0186 \\
\hline sum & 1.3918 & 1.3637 & 0.0206 & 1.6453 & 1.4968 & 0.0992 & 1.5818 & 1.4750 & 0.0724 & 1.5945 & 1.4698 & 0.0848 \\
\hline xargs. 1 & 1.3009 & 1.2179 & 0.0681 & 2.6305 & 1.5516 & 0.6953 & 2.2343 & 1.4268 & 0.5659 & 2.3135 & 1.4481 & 0.5976 \\
\hline \multicolumn{13}{|c|}{ The Large Corpus } \\
\hline$\overline{\text { E.coli }}$ & 1.2462 & 1.2462 & $<10^{-4}$ & 1.2322 & 1.2315 & 0.0006 & 1.2455 & 1.2450 & 0.0004 & 1.2428 & 1.2415 & 0.0011 \\
\hline & 1.2502 & 1.2502 & $<10^{-4}$ & 1.2341 & 1.2334 & 0.0006 & 1.2475 & 1.2469 & 0.0005 & 1.2448 & 1.2435 & 0.0010 \\
\hline & 1.2498 & 1.2498 & $<10^{-4}$ & 1.2313 & 1.2306 & 0.0006 & 1.2464 & 1.2458 & 0.0005 & 1.2434 & 1.2421 & 0.0011 \\
\hline & 1.2479 & 1.2479 & $<10^{-4}$ & 1.2330 & 1.2323 & 0.0006 & 1.2464 & 1.2457 & 0.0005 & 1.2437 & 1.2423 & 0.0011 \\
\hline & 1.2432 & 1.2432 & $<10^{-4}$ & 1.2310 & 1.2303 & 0.0006 & 1.2438 & 1.2434 & 0.0004 & 1.2413 & 1.2402 & 0.0008 \\
\hline & 1.2504 & 1.2504 & $<10^{-4}$ & 1.2391 & 1.2346 & 0.0037 & 1.2504 & 1.2466 & 0.0030 & 1.2481 & 1.2442 & 0.0032 \\
\hline bible.txt & 1.2186 & 1.2185 & 0.0001 & 1.2571 & 1.2456 & 0.0092 & 1.2681 & 1.2599 & 0.0065 & 1.2659 & 1.2555 & 0.0083 \\
\hline & 1.2304 & 1.2304 & $<10^{-4}$ & 1.2650 & 1.2540 & 0.0088 & 1.2793 & 1.2713 & 0.0063 & 1.2764 & 1.2661 & 0.0081 \\
\hline & 1.2561 & 1.2560 & 0.0001 & 1.2643 & 1.2536 & 0.0085 & 1.2923 & 1.2845 & 0.0061 & 1.2867 & 1.2768 & 0.0077 \\
\hline & 1.2375 & 1.2375 & $<10^{-4}$ & 1.2687 & 1.2581 & 0.0084 & 1.2846 & 1.2764 & 0.0064 & 1.2814 & 1.2716 & 0.0077 \\
\hline & 1.2657 & 1.2655 & 0.0002 & 1.2954 & 1.2752 & 0.0159 & 1.3158 & 1.3009 & 0.0114 & 1.3117 & 1.2943 & 0.0135 \\
\hline world192.txt & 1.2475 & 1.2471 & 0.0003 & 1.3134 & 1.2996 & 0.0107 & 1.3163 & 1.3061 & 0.0078 & 1.3158 & 1.3002 & 0.0119 \\
\hline & 1.2451 & 1.2449 & 0.0002 & 1.3186 & 1.3058 & 0.0098 & 1.3170 & 1.3075 & 0.0073 & 1.3173 & 1.3022 & 0.0116 \\
\hline & 1.2406 & 1.2403 & 0.0003 & 1.3375 & 1.3201 & 0.0132 & 1.3265 & 1.3133 & 0.0100 & 1.3287 & 1.3091 & 0.0150 \\
\hline \multicolumn{13}{|c|}{ The Calgary Corpus } \\
\hline bib & 1.2556 & 1.2542 & $\overline{0.0011}$ & 1.4610 & 1.3785 & 0.0598 & 1.4126 & 1.3504 & 0.0461 & 1.4223 & 1.3495 & 0.0539 \\
\hline book1 & 1.3272 & 1.3267 & 0.0004 & 1.3049 & 1.2920 & 0.0100 & 1.3553 & 1.3456 & 0.0073 & 1.3453 & 1.3320 & 0.0100 \\
\hline book2 & 1.2741 & 1.2736 & 0.0004 & 1.3502 & 1.3313 & 0.0142 & 1.3520 & 1.3378 & 0.0107 & 1.3517 & 1.3306 & 0.0158 \\
\hline geo & 1.5244 & 1.5197 & 0.0031 & 1.4653 & 1.4466 & 0.0129 & 1.5282 & 1.5146 & 0.0090 & 1.5156 & 1.5128 & 0.0018 \\
\hline news & 1.2966 & 1.2960 & 0.0004 & 1.4473 & 1.4253 & 0.0154 & 1.4202 & 1.4040 & 0.0115 & 1.4256 & 1.3966 & 0.0208 \\
\hline obj1 & 1.4453 & 1.4201 & 0.0177 & 1.6248 & 1.4867 & 0.0929 & 1.6017 & 1.5045 & 0.0646 & 1.6063 & 1.4921 & 0.0766 \\
\hline obj2 & 1.3444 & 1.3410 & 0.0025 & 1.5412 & 1.5212 & 0.0132 & 1.4971 & 1.4813 & 0.0107 & 1.5059 & 1.4627 & 0.0295 \\
\hline paper1 & 1.2486 & 1.2447 & 0.0032 & 1.6182 & 1.4536 & 0.1132 & 1.5075 & 1.3835 & 0.0897 & 1.5297 & 1.3860 & 0.1037 \\
\hline paper2 & 1.2843 & 1.2798 & 0.0036 & 1.4948 & 1.3775 & 0.0852 & 1.4544 & 1.3658 & 0.0649 & 1.4625 & 1.3640 & 0.0722 \\
\hline paper3 & 1.2840 & 1.2798 & 0.0032 & 1.5838 & 1.4161 & 0.1184 & 1.5113 & 1.3854 & 0.0909 & 1.5258 & 1.3879 & 0.0994 \\
\hline paper4 & 1.2801 & 1.2649 & 0.0120 & 1.9345 & 1.4833 & 0.3042 & 1.7521 & 1.4117 & 0.2411 & 1.7886 & 1.4242 & 0.2559 \\
\hline paper5 & 1.2828 & 1.2630 & 0.0156 & 1.9910 & 1.5193 & 0.3105 & 1.7860 & 1.4247 & 0.2536 & 1.8270 & 1.4345 & 0.2736 \\
\hline paper6 & 1.2402 & 1.2352 & 0.0040 & 1.6909 & 1.4752 & 0.1462 & 1.5523 & 1.3911 & 0.1159 & 1.5800 & 1.3963 & 0.1316 \\
\hline pic & 1.2570 & 1.2525 & 0.0036 & 1.2317 & 1.2005 & 0.0261 & 1.2821 & 1.2588 & 0.0185 & 1.2720 & 1.2488 & 0.0186 \\
\hline progc & 1.2577 & 1.2541 & 0.0028 & 1.6632 & 1.4758 & 0.1270 & 1.5448 & 1.4053 & 0.0993 & 1.5685 & 1.4064 & 0.1153 \\
\hline progl & 1.1912 & 1.1871 & 0.0035 & 1.5560 & 1.4023 & 0.1096 & 1.4408 & 1.3255 & 0.0870 & 1.4638 & 1.3348 & 0.0967 \\
\hline progp & 1.1599 & 1.1563 & 0.0032 & 1.6736 & 1.4681 & 0.1400 & 1.4929 & 1.3383 & 0.1155 & 1.5290 & 1.3550 & 0.1285 \\
\hline trans & 1.1370 & 1.1343 & 0.0024 & 1.6046 & 1.4754 & 0.0876 & 1.4264 & 1.3291 & 0.0732 & 1.4621 & 1.3509 & 0.0823 \\
\hline
\end{tabular}

Table 6: Upper bounds $c_{A}$ on the performance ratios achieved by $A \in\{M T F, T S, B I T, C O M B\}$ as implied by Theorems $1-4$. The values are compared to the experimentally observed competitiveness $c_{A}^{*}$, which is the actual cost incurred by $A$ to that of the pairwise optimum. The comparison yields a relative error $f_{c_{A}}$. 


\begin{tabular}{|c|c|c|c|c|c|}
\hline file & $\lambda$ & $c_{M T F}^{\lambda}$ & $f_{c_{M T F}^{\lambda}}$ & $c_{B I T}^{\lambda}$ & $f_{c_{B I T}^{\lambda}}$ \\
\hline \multicolumn{6}{|c|}{ The Canterbury Corpus } \\
\hline alice29.txt & 0.3898 & 1.4391 & 0.1244 & 1.7195 & 0.2833 \\
\hline asyoulik.txt & 0.3786 & 1.4508 & 0.1093 & 1.7254 & 0.2664 \\
\hline cp.html & 0.5072 & 1.3270 & 0.0343 & 1.6635 & 0.1736 \\
\hline fields.c & 0.7109 & 1.1689 & 0.0025 & 1.5845 & 0.1440 \\
\hline grammar.lsp & 0.6802 & 1.1903 & 0.0226 & 1.5952 & 0.1393 \\
\hline kennedy.xls & 0.0782 & 1.8549 & 0.0349 & 1.7500 & 0.2072 \\
\hline & 0.0896 & 1.8355 & 0.0638 & 1.7500 & 0.2272 \\
\hline lcet10.txt & 0.3664 & 1.4637 & 0.1466 & 1.7319 & 0.3062 \\
\hline plrabn12.txt & 0.3226 & 1.5122 & 0.1419 & 1.7500 & 0.2975 \\
\hline ptt5 & 0.2915 & 1.5486 & 0.2364 & 1.7500 & 0.3902 \\
\hline sum & 0.4165 & 1.4120 & 0.0354 & 1.7060 & 0.1566 \\
\hline xargs.1 & 0.6328 & 1.2249 & 0.0057 & 1.6125 & 0.1301 \\
\hline \multicolumn{6}{|c|}{ The Large Corpus } \\
\hline \multirow[t]{6}{*}{ E.coli } & 0.3169 & $\begin{array}{c}1.5188 \\
\end{array}$ & 0.2187 & 1.7500 & 0.4056 \\
\hline & 0.3140 & 1.5220 & 0.2174 & 1.7500 & 0.4035 \\
\hline & 0.3120 & 1.5244 & 0.2197 & 1.7500 & 0.4047 \\
\hline & 0.3159 & 1.5199 & 0.2180 & 1.7500 & 0.4048 \\
\hline & 0.3195 & 1.5157 & 0.2192 & 1.7500 & 0.4074 \\
\hline & 0.3175 & 1.5180 & 0.2140 & 1.7500 & 0.4038 \\
\hline \multirow[t]{5}{*}{ bible.txt } & 0.3679 & 1.4621 & 0.1998 & 1.7310 & 0.3740 \\
\hline & 0.3631 & 1.4672 & 0.1925 & 1.7336 & 0.3637 \\
\hline & 0.3363 & 1.4967 & 0.1916 & 1.7483 & 0.3611 \\
\hline & 0.3589 & 1.4718 & 0.1894 & 1.7359 & 0.3600 \\
\hline & 0.3494 & 1.4821 & 0.1711 & 1.7410 & 0.3383 \\
\hline \multirow[t]{3}{*}{ world192.txt } & 0.3868 & 1.4421 & 0.1564 & 1.7211 & 0.3177 \\
\hline & 0.3936 & 1.4351 & 0.1528 & 1.7176 & 0.3137 \\
\hline & 0.4111 & 1.4173 & 0.1428 & 1.7087 & 0.3010 \\
\hline \multicolumn{6}{|c|}{ The Calgary Corpus } \\
\hline bib & 0.4547 & 1.3749 & 0.0962 & 1.6874 & 0.2496 \\
\hline book1 & 0.3104 & 1.5263 & 0.1504 & 1.7500 & 0.3006 \\
\hline book2 & 0.3853 & 1.4438 & 0.1336 & 1.7219 & 0.2871 \\
\hline geo & 0.2969 & 1.5422 & 0.0148 & 1.7500 & 0.1554 \\
\hline news & 0.4282 & 1.4004 & 0.0805 & 1.7002 & 0.2109 \\
\hline obj1 & 0.3840 & 1.4451 & 0.0176 & 1.7225 & 0.1450 \\
\hline obj2 & 0.4424 & 1.3866 & 0.0340 & 1.6933 & 0.1431 \\
\hline paper1 & 0.5208 & 1.3151 & 0.0565 & 1.6575 & 0.1981 \\
\hline paper2 & 0.4351 & 1.3937 & 0.0890 & 1.6968 & 0.2423 \\
\hline paper3 & 0.4702 & 1.3604 & 0.0629 & 1.6802 & 0.2128 \\
\hline paper4 & 0.5585 & 1.2833 & 0.0145 & 1.6416 & 0.1628 \\
\hline paper5 & 0.5710 & 1.2731 & 0.0080 & 1.6366 & 0.1487 \\
\hline paper6 & 0.5512 & 1.2893 & 0.0437 & 1.6446 & 0.1823 \\
\hline pic & 0.2915 & 1.5486 & 0.2364 & 1.7500 & 0.3902 \\
\hline progc & 0.5291 & 1.3080 & 0.0429 & 1.6540 & 0.1770 \\
\hline progl & 0.5594 & 1.2826 & 0.0804 & 1.6413 & 0.2382 \\
\hline progp & 0.6433 & 1.2171 & 0.0526 & 1.6085 & 0.2019 \\
\hline trans & 0.6652 & 1.2011 & 0.0588 & 1.6005 & 0.2042 \\
\hline
\end{tabular}

Table 7: Competitive ratios $c_{M T F}^{\lambda}$ and $c_{B I T}^{\lambda}$ according to Corollaries 1 and 2. The values are compared to $c_{M T F}^{*}$ and $c_{B I T}^{*}$, respectively, yielding relative errors $f_{c_{M T F}^{\lambda}}$ and $f_{c_{B I T}^{\lambda}}$. 


\section{Appendix B}

\begin{tabular}{|c|c|c|c|c|c|c|c|c|}
\hline file & seq. size & diff. pages & $s / r$ & $l / r$ & $l_{p} / l$ & $l_{i} / l$ & $l_{c} / l$ & $\lambda$ \\
\hline bzip2 g7 & 10481530 & 353 & 0.4871 & 0.5129 & 0.5017 & 0.4983 & 0.5584 & 0.2864 \\
\hline bzip2 g9 & 10481504 & 825 & 0.4715 & 0.5285 & 0.4760 & 0.5240 & 0.5819 & 0.3076 \\
\hline bzip2 p7 & 10481167 & 346 & 0.4790 & 0.5210 & 0.4866 & 0.5134 & 0.5742 & 0.2992 \\
\hline bzip2 p9 & 10481213 & 826 & 0.4641 & 0.5359 & 0.4628 & 0.5372 & 0.5957 & 0.3193 \\
\hline bzip2 s7 & 10481540 & 340 & 0.4819 & 0.5181 & 0.4935 & 0.5065 & 0.5675 & 0.2940 \\
\hline bzip2 s9 & 10481386 & 826 & 0.4663 & 0.5337 & 0.4677 & 0.5323 & 0.5903 & 0.3151 \\
\hline crafty & 10481629 & 1007 & 0.3199 & 0.6801 & 0.2525 & 0.7475 & 0.7712 & 0.5245 \\
\hline eon cook & 10481801 & 533 & 0.4976 & 0.5024 & 0.3749 & 0.6251 & 0.7495 & 0.3766 \\
\hline eon kajiya & 10482257 & 397 & 0.5114 & 0.4886 & 0.3721 & 0.6279 & 0.7776 & 0.3800 \\
\hline eon rush & 10482256 & 374 & 0.5067 & 0.4933 & 0.3766 & 0.6234 & 0.7678 & 0.3788 \\
\hline gap & 10481513 & 901 & 0.4011 & 0.5989 & 0.3965 & 0.6035 & 0.6417 & 0.3843 \\
\hline $\operatorname{gcc} 166$ & 10481586 & 706 & 0.3663 & 0.6337 & 0.3676 & 0.6324 & 0.6749 & 0.4277 \\
\hline $\operatorname{gcc} 200$ & 10479248 & 542 & 0.3438 & 0.6562 & 0.3333 & 0.6667 & 0.7002 & 0.4595 \\
\hline gcc expr & 10481541 & 778 & 0.3557 & 0.6443 & 0.3527 & 0.6473 & 0.6871 & 0.4427 \\
\hline gcc integ & 10481491 & 716 & 0.3573 & 0.6427 & 0.3546 & 0.6454 & 0.6857 & 0.4407 \\
\hline gcc scilab & 10481568 & 809 & 0.3510 & 0.6490 & 0.3357 & 0.6643 & 0.7018 & 0.4555 \\
\hline gzip graphic & 10481443 & 576 & 0.4377 & 0.5623 & 0.4566 & 0.5434 & 0.5933 & 0.3336 \\
\hline gzip log & 10481298 & 429 & 0.4871 & 0.5129 & 0.5680 & 0.4320 & 0.4607 & 0.2363 \\
\hline gzip program & 10481317 & 509 & 0.4302 & 0.5698 & 0.4706 & 0.5294 & 0.5681 & 0.3237 \\
\hline gzip random & 10480337 & 526 & 0.4320 & 0.5680 & 0.4565 & 0.5435 & 0.5927 & 0.3367 \\
\hline gzip source & 10481850 & 482 & 0.4402 & 0.5598 & 0.4920 & 0.5080 & 0.5420 & 0.3034 \\
\hline $\mathrm{mcf}$ & 10481598 & 3003 & 0.2487 & 0.7513 & 0.2436 & 0.7564 & 0.7713 & 0.5794 \\
\hline parser & 10481694 & 1353 & 0.4209 & 0.5791 & 0.3811 & 0.6189 & 0.6634 & 0.3841 \\
\hline perl diffmail & 10481359 & 996 & 0.3354 & 0.6646 & 0.3044 & 0.6956 & 0.7326 & 0.4869 \\
\hline perl makerand & 10482174 & 3151 & 0.3296 & 0.6704 & 0.1901 & 0.8099 & 0.8142 & 0.5459 \\
\hline perl perfect & 10481539 & 580 & 0.3172 & 0.6828 & 0.2808 & 0.7192 & 0.7553 & 0.5157 \\
\hline perl splitmail & 10481588 & 702 & 0.3332 & 0.6668 & 0.3015 & 0.6985 & 0.7353 & 0.4903 \\
\hline twolf & 10481015 & 469 & 0.3467 & 0.6533 & 0.3148 & 0.6852 & 0.7190 & 0.4697 \\
\hline vortex one & 10481880 & 1589 & 0.3629 & 0.6371 & 0.2420 & 0.7580 & 0.7839 & 0.4994 \\
\hline vortex two & 10481864 & 1647 & 0.3603 & 0.6397 & 0.2393 & 0.7607 & 0.7864 & 0.5031 \\
\hline vortex three & 10481875 & 1642 & 0.3585 & 0.6415 & 0.2369 & 0.7631 & 0.7888 & 0.5060 \\
\hline vpr place & 10481572 & 782 & 0.4098 & 0.5902 & 0.4591 & 0.5409 & 0.5624 & 0.3319 \\
\hline vpr route & 10481581 & 605 & 0.4476 & 0.5524 & 0.4256 & 0.5744 & 0.6254 & 0.3455 \\
\hline ammp & 10481899 & 251 & 0.4102 & 0.5898 & 0.4050 & 0.5950 & 0.6541 & 0.3858 \\
\hline applu & 10482411 & 1394 & 0.3156 & 0.6844 & 0.2410 & 0.7590 & 0.7640 & 0.5229 \\
\hline apsi & 10474135 & 517 & 0.3426 & 0.6574 & 0.2804 & 0.7196 & 0.7448 & 0.4896 \\
\hline art & 10481866 & 1133 & 0.3962 & 0.6038 & 0.4240 & 0.5760 & 0.5997 & 0.3621 \\
\hline equake & 10481760 & 747 & 0.5088 & 0.4912 & 0.5480 & 0.4520 & 0.5346 & 0.2626 \\
\hline facerec & 10481552 & 527 & 0.4081 & 0.5919 & 0.4130 & 0.5870 & 0.6360 & 0.3765 \\
\hline fma3d & 10479601 & 520 & 0.4946 & 0.5054 & 0.5527 & 0.4473 & 0.4821 & 0.2436 \\
\hline galgel & 10481912 & 516 & 0.5967 & 0.4033 & 0.4931 & 0.5069 & 0.5618 & 0.2266 \\
\hline lucas & 10481561 & 1570 & 0.4984 & 0.5016 & 0.4829 & 0.5171 & 0.5266 & 0.2641 \\
\hline mesa & 10482000 & 1398 & 0.6044 & 0.3956 & 0.5280 & 0.4720 & 0.4774 & 0.1889 \\
\hline mgrid & 10482496 & 1977 & 0.4200 & 0.5800 & 0.3325 & 0.6675 & 0.6741 & 0.3909 \\
\hline sixtrack & 10481307 & 1668 & 0.4867 & 0.5133 & 0.4811 & 0.5189 & 0.5300 & 0.2720 \\
\hline swim & 10481645 & 2098 & 0.3741 & 0.6259 & 0.4067 & 0.5933 & 0.6188 & 0.3872 \\
\hline wupwise & 10481968 & 569 & 0.3581 & 0.6419 & 0.3062 & 0.6938 & 0.7229 & 0.4640 \\
\hline
\end{tabular}

Table 8: Characteristics of the memory access traces. Length of the sequence, number of different pages requested and statistics about the runs. 


\begin{tabular}{|c|c|c|c|c|c|c|c|c|c|c|}
\hline file & $O P T^{*}$ & $f_{O P T}$ & $M T F^{*}$ & $f_{M T F}$ & $T S^{*}$ & $f_{T S}$ & $B I T^{*}$ & $f_{B I T}$ & $C O M B^{*}$ & $f_{C O M B}$ \\
\hline bzip2 g7 & 19041305 & 0.0005 & 23808453 & $\overline{0}$ & 23750319 & 0.0045 & 23959189.9375 & $\overline{0.0003}$ & 23753803.9375 & $\overline{0.0075}$ \\
\hline bzip2 g9 & 19595974 & 0.0034 & 24482053 & 0 & 24708075 & 0.0217 & 24638102.3750 & 0.0197 & 24747882.5000 & 0.0161 \\
\hline bzip2 p7 & 19119813 & 0.0004 & 23800332 & 0 & 23948967 & 0.0044 & 23914290.3125 & 0.0056 & 23881583.7500 & 0.0070 \\
\hline bzip2 p9 & 19651460 & 0.0033 & 24441294 & 0 & 24859257 & 0.0221 & 24643607.8125 & 0.0221 & 24697333.6875 & 0.0216 \\
\hline bzip2 s7 & 19072205 & 0.0003 & 23779070 & 0 & 23830100 & 0.0043 & 23879476.6875 & 0.0042 & 23825573.8750 & 0.0061 \\
\hline bzip2 s9 & 19625107 & 0.0034 & 24447705 & 0 & 24800612 & 0.0218 & 24724719.9375 & 0.0175 & 24791288.1250 & 0.0163 \\
\hline crafty & 27435162 & 0.0022 & 32797854 & 0 & 40157785 & 0.0220 & 36614133.6250 & 0.0182 & 36696794.4375 & 0.0364 \\
\hline eon cook & 18340223 & 0.0014 & 21921452 & 0 & 23311621 & 0.0099 & 22278605.8750 & 0.0074 & 22444844.0000 & 0.0097 \\
\hline eon kajiya & 17994231 & 0.0009 & 21377727 & 0 & 22718353 & 0.0055 & 21662773.6875 & 0.0035 & 21878515.6250 & 0.0037 \\
\hline eon rush & 18055725 & 0.0008 & 21477451 & 0 & 22794425 & 0.0049 & 21731393.9375 & 0.0053 & 21956685.3125 & 0.0047 \\
\hline gap & 23116558 & 0.0033 & 28791857 & 0 & 30930257 & 0.0211 & 30213037.6875 & 0.0164 & 30213024.0625 & 0.0222 \\
\hline gcc 166 & 26454187 & 0.0016 & 32890776 & 0 & 36572110 & 0.0112 & 35253853.1250 & 0.0100 & 35417589.0000 & 0.0131 \\
\hline $\operatorname{gcc} 200$ & 26383889 & 0.0010 & 32290397 & 0 & 37023743 & 0.0066 & 35163962.5625 & 0.0054 & 35338105.2500 & 0.0113 \\
\hline gcc expr & 26633066 & 0.0022 & 32902894 & 0 & 37085519 & 0.0131 & 35523302.3750 & 0.0113 & 35643474.0000 & 0.0171 \\
\hline gcc integ & 26721936 & 0.0017 & 33055848 & 0 & 37202802 & 0.0112 & 35646241.1250 & 0.0102 & 35795867.1250 & 0.0150 \\
\hline gcc scilab & 26224155 & 0.0019 & 32165133 & 0 & 36679428 & 0.0151 & 34901247.1875 & 0.0116 & 34971861.3125 & 0.0206 \\
\hline gzip graphic & 20977689 & 0.0009 & 26272194 & 0 & 26938644 & 0.0109 & 26888501.5000 & 0.0090 & 26759993.5000 & 0.0146 \\
\hline gzip log & 16265504 & 0.0004 & 19883978 & 0 & 19148234 & 0.0089 & 19761100.0000 & 0.0056 & 19500459.8750 & 0.0134 \\
\hline gzip program & 20323992 & 0.0005 & 25396805 & 0 & 25800601 & 0.0092 & 26019709.0625 & 0.0061 & 25824171.1875 & 0.0127 \\
\hline gzip random & 21990881 & 0.0005 & 27749992 & 0 & 28506825 & 0.0089 & 28555206.1875 & 0.0049 & 28307865.8125 & 0.0142 \\
\hline gzip source & 19305104 & 0.0004 & 24065375 & 0 & 24093040 & 0.0091 & 24424265.2500 & 0.0082 & 24243553.8125 & 0.0131 \\
\hline mcf & 47240992 & 0.0110 & 57523649 & 0 & 74415158 & 0.1072 & 67398608.1250 & 0.0904 & 66914378.2500 & 0.1249 \\
\hline parser & 20951524 & 0.0073 & 25770547 & 0 & 27559490 & 0.0544 & 26735276.7500 & 0.0433 & 26805792.6250 & 0.0492 \\
\hline perl diffmail & 26608785 & 0.0019 & 32269697 & 0 & 37719043 & 0.0236 & 35334990.3125 & 0.0203 & 35657725.0625 & 0.0254 \\
\hline perl makerand & 22763892 & 0.0117 & 27474091 & 0 & 32428760 & 0.2896 & 29487743.8750 & 0.2409 & 30123287.3125 & 0.2495 \\
\hline perl perfect & 28207213 & 0.0006 & 33898407 & 0 & 40845230 & 0.0073 & 37799755.3750 & 0.0050 & 38288086.5000 & 0.0086 \\
\hline perl splitmail & 27771215 & 0.0011 & 33718801 & 0 & 39781745 & 0.0107 & 37130853.0625 & 0.0094 & 37484745.6875 & 0.0144 \\
\hline twolf & 25405630 & 0.0007 & 30805751 & 0 & 35694923 & 0.0051 & 33581876.6250 & 0.0041 & 34062627.7500 & 0.0026 \\
\hline vortex one & 27623852 & 0.0051 & 33569584 & 0 & 40733985 & 0.0549 & 36794640.1250 & 0.0465 & 37343411.5625 & 0.0550 \\
\hline vortex two & 27735820 & 0.0050 & 33692025 & 0 & 40946422 & 0.0593 & 37016741.3750 & 0.0486 & 37500413.4375 & 0.0594 \\
\hline vortex three & 27807823 & 0.0043 & 33764481 & 0 & 41092298 & 0.0596 & 37094391.6250 & 0.0497 & 37664598.8750 & 0.0582 \\
\hline vpr place & 23362831 & 0.0010 & 29937974 & 0 & 30660466 & 0.0183 & 30914425.8125 & 0.0149 & 30650092.8125 & 0.0226 \\
\hline vpr route & 20484025 & 0.0009 & 25410421 & 0 & 26525432 & 0.0123 & 26063059.5000 & 0.0091 & 26000790.5000 & 0.0158 \\
\hline ammp & 21137925 & $<10^{-4}$ & 25873293 & 0 & 27571783 & 0.0022 & 26974465.7500 & 0.0020 & 27132639.3750 & 0.0007 \\
\hline applu & 17402848 & 0.0005 & 19857152 & 0 & 22578118 & 0.0853 & 21244261.1250 & 0.0686 & 21447265.0625 & 0.0753 \\
\hline apsi & 20282849 & 0.0002 & 23680360 & 0 & 27411563 & 0.0094 & 25593928.0000 & 0.0091 & 25805319.8125 & 0.0151 \\
\hline art & 19928722 & 0.0005 & 24635070 & 0 & 25379249 & 0.0498 & 25510315.3750 & 0.0343 & 25364097.3125 & 0.0423 \\
\hline equake & 16962520 & 0.0010 & 20879076 & 0 & 20147541 & 0.0259 & 20590410.0000 & 0.0192 & 20445848.3750 & 0.0234 \\
\hline facerec & 21395553 & 0.0008 & 26375120 & 0 & 27938951 & 0.0087 & 27440575.9375 & 0.0082 & 27505873.6875 & 0.0095 \\
\hline fma3d & 17865239 & 0.0002 & 22430936 & 0 & 21664281 & 0.0121 & 22241428.3750 & 0.0088 & 22043067.6875 & 0.0133 \\
\hline galgel & 17136944 & 0.0003 & 21405539 & 0 & 21347139 & 0.0121 & 20824361.2500 & 0.0094 & 20708296.8750 & 0.0207 \\
\hline lucas & 16872346 & 0.0089 & 21045514 & 0 & 20465514 & 0.1053 & 20702051.8125 & 0.0794 & 20606735.4375 & 0.0870 \\
\hline mesa & 18514269 & 0.0004 & 24643701 & 0 & 23352719 & 0.0830 & 23283006.1250 & 0.0629 & 23050166.8125 & 0.0784 \\
\hline mgrid & 17736426 & 0.0002 & 21757472 & 0 & 22386216 & 0.1741 & 21980486.9375 & 0.1321 & 22129566.2500 & 0.1371 \\
\hline sixtrack & 31058863 & 0.0046 & 43390757 & 0 & 43099241 & 0.0577 & 43264831.9375 & 0.0443 & 43256346.0625 & 0.0464 \\
\hline swim & 27568308 & 0.0042 & 35908171 & 0 & 37284035 & 0.1117 & 37237966.3750 & 0.0918 & 37451208.6875 & 0.0898 \\
\hline wupwise & 16766967 & 0.0007 & 19107193 & 0 & 21169868 & 0.0140 & 20246223.3750 & 0.0096 & 20232872.6250 & 0.0204 \\
\hline
\end{tabular}

Table 9: Actual service costs incurred by the algorithms on the memory access traces. Comparison of the actual costs to the bounds of Lemmas 1-5 yields the relative errors $f_{A}$, for the various strategies $A$. 


\begin{tabular}{|c|c|c|c|c|c|}
\hline file & $\overline{O P T^{*}}$ & $\overline{M T F^{*}}$ & $\overline{T S^{*}}$ & $\overline{B I T^{*}}$ & $\overline{C O M B^{*}}$ \\
\hline bzip2 g7 & 1.8167 & 2.2715 & 2.2659 & 2.2858 & 2.2663 \\
\hline bzip2 g9 & 1.8696 & 2.3357 & 2.3573 & 2.3506 & 2.3611 \\
\hline bzip2 p7 & 1.8242 & 2.2708 & 2.2850 & 2.2816 & 2.2785 \\
\hline bzip2 p9 & 1.8749 & 2.3319 & 2.3718 & 2.3512 & 2.3563 \\
\hline bzip2 s7 & 1.8196 & 2.2687 & 2.2735 & 2.2782 & 2.2731 \\
\hline bzip2 s9 & 1.8724 & 2.3325 & 2.3662 & 2.3589 & 2.3653 \\
\hline crafty & 2.6175 & 3.1291 & 3.8313 & 3.4932 & 3.5011 \\
\hline eon cook & 1.7497 & 2.0914 & 2.2240 & 2.1255 & 2.1413 \\
\hline eon kajiya & 1.7166 & 2.0394 & 2.1673 & 2.0666 & 2.0872 \\
\hline eon rush & 1.7225 & 2.0489 & 2.1746 & 2.0732 & 2.0947 \\
\hline gap & 2.2055 & 2.7469 & 2.9509 & 2.8825 & 2.8825 \\
\hline gcc 166 & 2.5239 & 3.1380 & 3.4892 & 3.3634 & 3.3790 \\
\hline gcc 200 & 2.5177 & 3.0814 & 3.5331 & 3.3556 & 3.3722 \\
\hline gcc expr & 2.5409 & 3.1391 & 3.5382 & 3.3891 & 3.4006 \\
\hline gcc integ & 2.5494 & 3.1537 & 3.5494 & 3.4009 & 3.4152 \\
\hline gcc scilab & 2.5019 & 3.0687 & 3.4994 & 3.3298 & 3.3365 \\
\hline gzip graphic & 2.0014 & 2.5065 & 2.5701 & 2.5653 & 2.5531 \\
\hline gzip log & 1.5519 & 1.8971 & 1.8269 & 1.8854 & 1.8605 \\
\hline gzip program & 1.9391 & 2.4231 & 2.4616 & 2.4825 & 2.4638 \\
\hline gzip random & 2.0983 & 2.6478 & 2.7200 & 2.7246 & 2.7010 \\
\hline gzip source & 1.8418 & 2.2959 & 2.2985 & 2.3301 & 2.3129 \\
\hline mcf & 4.5070 & 5.4881 & 7.0996 & 6.4302 & 6.3840 \\
\hline parser & 1.9989 & 2.4586 & 2.6293 & 2.5507 & 2.5574 \\
\hline perl diffmail & 2.5387 & 3.0788 & 3.5987 & 3.3712 & 3.4020 \\
\hline perl makerand & 2.1717 & 2.6210 & 3.0937 & 2.8131 & 2.8738 \\
\hline perl perfect & 2.6911 & 3.2341 & 3.8969 & 3.6063 & 3.6529 \\
\hline perl splitmail & 2.6495 & 3.2170 & 3.7954 & 3.5425 & 3.5762 \\
\hline twolf & 2.4240 & 2.9392 & 3.4057 & 3.2041 & 3.2499 \\
\hline vortex one & 2.6354 & 3.2026 & 3.8861 & 3.5103 & 3.5627 \\
\hline vortex two & 2.6461 & 3.2143 & 3.9064 & 3.5315 & 3.5776 \\
\hline vortex three & 2.6529 & 3.2212 & 3.9203 & 3.5389 & 3.5933 \\
\hline vpr place & 2.2289 & 2.8562 & 2.9252 & 2.9494 & 2.9242 \\
\hline vpr route & 1.9543 & 2.4243 & 2.5307 & 2.4866 & 2.4806 \\
\hline ammp & 2.0166 & 2.4684 & 2.6304 & 2.5734 & 2.5885 \\
\hline applu & 1.6602 & 1.8943 & 2.1539 & 2.0267 & 2.046 \\
\hline apsi & 1.9365 & 2.2608 & 2.6171 & 2.4435 & 2.4637 \\
\hline art & 1.9013 & 2.3503 & 2.4213 & 2.4338 & 2.4198 \\
\hline equake & 1.6183 & 1.9919 & 1.9222 & 1.9644 & 1.9506 \\
\hline facerec & 2.0413 & 2.5163 & 2.6655 & 2.6180 & 2.6242 \\
\hline fma3d & 1.7048 & 2.1404 & 2.0673 & 2.1224 & 2.1034 \\
\hline galgel & 1.6349 & 2.0421 & 2.0366 & 1.9867 & 1.9756 \\
\hline lucas & 1.6097 & 2.0079 & 1.9525 & 1.9751 & 1.9660 \\
\hline mesa & 1.7663 & 2.3510 & 2.2279 & 2.2212 & 2.1990 \\
\hline mgrid & 1.6920 & 2.0756 & 2.1356 & 2.0969 & 2.1111 \\
\hline sixtrack & 2.9633 & 4.1398 & 4.1120 & 4.1278 & 4.1270 \\
\hline swim & 2.6302 & 3.4258 & 3.5571 & 3.5527 & 3.5730 \\
\hline wupwise & 1.5996 & 1.8229 & 2.0196 & 1.9315 & 1.9303 \\
\hline
\end{tabular}

Table 10: Average service cost per request incurred by the algorithms on the memory access traces. 


\begin{tabular}{|c|c|c|c|c|c|c|c|c|c|c|c|c|}
\hline file & $c_{M T F}$ & $c_{M T F}^{*}$ & $f_{c_{M T F}}$ & $c_{T S}$ & $c_{T S}^{*}$ & $f_{c_{T S}}$ & $c_{B I T}$ & $c_{B I T}^{*}$ & $f_{c_{B I T}}$ & $c_{C O M B}$ & $c_{C O M B}^{*}$ & $f_{C_{C O M B}}$ \\
\hline bzip2 g7 & 1.2510 & 1.2504 & 0.0005 & 1.2535 & 1.2473 & 0.0050 & 1.2585 & 1.2583 & 0.0002 & 1.2575 & 1.2475 & 0.0080 \\
\hline bzip2 g9 & 1.2544 & 1.2493 & 0.0040 & 1.2935 & 1.2609 & 0.0259 & 1.2869 & 1.2573 & 0.0236 & 1.2882 & 1.2629 & 0.0201 \\
\hline bzip2 p7 & 1.2453 & 1.2448 & 0.0004 & 1.2586 & 1.2526 & 0.0048 & 1.2582 & 1.2508 & 0.0060 & 1.2583 & 1.2490 & 0.0074 \\
\hline bzip2 p9 & 1.2487 & 1.2437 & 0.0040 & 1.2980 & 1.2650 & 0.0261 & 1.2865 & 1.2540 & 0.0259 & 1.2888 & 1.2568 & 0.0255 \\
\hline bzip2 s7 & 1.2473 & 1.2468 & 0.0004 & 1.2553 & 1.2495 & 0.0047 & 1.2578 & 1.2521 & 0.0046 & 1.2573 & 1.2492 & 0.0065 \\
\hline bzip2 s9 & 1.2506 & 1.2457 & 0.0039 & 1.2963 & 1.2637 & 0.0258 & 1.2867 & 1.2599 & 0.0213 & 1.2886 & 1.2632 & 0.0201 \\
\hline crafty & 1.1982 & 1.1955 & 0.0023 & 1.4994 & 1.4637 & 0.0244 & 1.3620 & 1.3346 & 0.0205 & 1.3895 & 1.3376 & 0.0388 \\
\hline eon cook & 1.1971 & 1.1953 & 0.0016 & 1.2857 & 1.2711 & 0.0115 & 1.2255 & 1.2147 & 0.0089 & 1.2376 & 1.2238 & 0.0112 \\
\hline eon kajiya & 1.1893 & 1.1880 & 0.0010 & 1.2708 & 1.2625 & 0.0065 & 1.2092 & 1.2039 & 0.0045 & 1.2215 & 1.2159 & 0.0047 \\
\hline eon rush & 1.1905 & 1.1895 & 0.0008 & 1.2697 & 1.2624 & 0.0058 & 1.2110 & 1.2036 & 0.0062 & 1.2227 & 1.2161 & 0.0055 \\
\hline gap & 1.2502 & 1.2455 & 0.0037 & 1.3714 & 1.3380 & 0.0250 & 1.3331 & 1.3070 & 0.0200 & 1.3408 & 1.3070 & 0.0259 \\
\hline gcc 166 & 1.2455 & 1.2433 & 0.0018 & 1.4004 & 1.3825 & 0.0130 & 1.3483 & 1.3326 & 0.0118 & 1.3587 & 1.3388 & 0.0149 \\
\hline $\operatorname{gcc} 200$ & 1.2251 & 1.2239 & 0.0010 & 1.4139 & 1.4033 & 0.0076 & 1.3414 & 1.3328 & 0.0064 & 1.3559 & 1.3394 & 0.0123 \\
\hline gcc expr & 1.2383 & 1.2354 & 0.0023 & 1.4140 & 1.3925 & 0.0155 & 1.3520 & 1.3338 & 0.0136 & 1.3644 & 1.3383 & 0.0195 \\
\hline gcc integ & 1.2394 & 1.2370 & 0.0019 & 1.4105 & 1.3922 & 0.0131 & 1.3500 & 1.3340 & 0.0120 & 1.3621 & 1.3396 & 0.0168 \\
\hline gcc scilab & 1.2290 & 1.2265 & 0.0020 & 1.4226 & 1.3987 & 0.0171 & 1.3490 & 1.3309 & 0.0136 & 1.3637 & 1.3336 & 0.0226 \\
\hline gzip graphic & 1.2537 & 1.2524 & 0.0011 & 1.2996 & 1.2842 & 0.0120 & 1.2946 & 1.2818 & 0.0100 & 1.2956 & 1.2756 & 0.0156 \\
\hline gzip log & 1.2230 & 1.2225 & 0.0004 & 1.1883 & 1.1772 & 0.0094 & 1.2222 & 1.2149 & 0.0060 & 1.2154 & 1.1989 & 0.0138 \\
\hline gzip program & 1.2505 & 1.2496 & 0.0007 & 1.2821 & 1.2695 & 0.0100 & 1.2889 & 1.2802 & 0.0068 & 1.2876 & 1.2706 & 0.0133 \\
\hline gzip random & 1.2627 & 1.2619 & 0.0007 & 1.3086 & 1.2963 & 0.0095 & 1.3057 & 1.2985 & 0.0055 & 1.3063 & 1.2873 & 0.0148 \\
\hline gzip source & 1.2473 & 1.2466 & 0.0006 & 1.2601 & 1.2480 & 0.0096 & 1.2762 & 1.2652 & 0.0087 & 1.2729 & 1.2558 & 0.0136 \\
\hline $\operatorname{mcf}$ & 1.2322 & 1.2177 & 0.0119 & 1.7648 & 1.5752 & 0.1204 & 1.5738 & 1.4267 & 0.1031 & 1.6120 & 1.4164 & 0.1381 \\
\hline parser & 1.2401 & 1.2300 & 0.0082 & 1.3984 & 1.3154 & 0.0631 & 1.3418 & 1.2761 & 0.0515 & 1.3531 & 1.2794 & 0.0576 \\
\hline perl diffmail & 1.2153 & 1.2127 & 0.0021 & 1.4540 & 1.4175 & 0.0257 & 1.3576 & 1.3279 & 0.0223 & 1.3769 & 1.3401 & 0.0275 \\
\hline perl makerand & 1.2217 & 1.2069 & 0.0122 & 1.8596 & 1.4246 & 0.3054 & 1.6269 & 1.2954 & 0.2560 & 1.6735 & 1.3233 & 0.2646 \\
\hline perl perfect & 1.2026 & 1.2018 & 0.0007 & 1.4597 & 1.4480 & 0.0080 & 1.3477 & 1.3401 & 0.0057 & 1.3701 & 1.3574 & 0.0093 \\
\hline perl splitmail & 1.2158 & 1.2142 & 0.0013 & 1.4498 & 1.4325 & 0.0121 & 1.3513 & 1.3370 & 0.0107 & 1.3710 & 1.3498 & 0.0157 \\
\hline twolf & 1.2134 & 1.2126 & 0.0007 & 1.4132 & 1.4050 & 0.0058 & 1.3281 & 1.3218 & 0.0048 & 1.3451 & 1.3408 & 0.0033 \\
\hline vortex one & 1.2218 & 1.2152 & 0.0054 & 1.5640 & 1.4746 & 0.0607 & 1.4013 & 1.3320 & 0.0521 & 1.4339 & 1.3519 & 0.0607 \\
\hline vortex two & 1.2212 & 1.2147 & 0.0053 & 1.5722 & 1.4763 & 0.0650 & 1.4068 & 1.3346 & 0.0541 & 1.4399 & 1.3521 & 0.0650 \\
\hline vortex three & 1.2198 & 1.2142 & 0.0046 & 1.5730 & 1.4777 & 0.0645 & 1.4065 & 1.3340 & 0.0544 & 1.4398 & 1.3545 & 0.0630 \\
\hline vpr place & 1.2829 & 1.2814 & 0.0011 & 1.3378 & 1.3124 & 0.0194 & 1.3444 & 1.3232 & 0.0160 & 1.3431 & 1.3119 & 0.0237 \\
\hline vpr route & 1.2417 & 1.2405 & 0.0009 & 1.3121 & 1.2949 & 0.0133 & 1.2851 & 1.2724 & 0.0100 & 1.2905 & 1.2693 & 0.0167 \\
\hline ammp & 1.2241 & 1.2240 & $<10^{-4}$ & 1.3073 & 1.3044 & 0.0023 & 1.2788 & 1.2761 & 0.0021 & 1.2845 & 1.2836 & 0.0007 \\
\hline applu & 1.1419 & 1.1410 & 0.0008 & 1.4092 & 1.2974 & 0.0862 & 1.3054 & 1.2207 & 0.0693 & 1.3261 & 1.2324 & 0.0760 \\
\hline apsi & 1.1679 & 1.1675 & 0.0003 & 1.3646 & 1.3515 & 0.0097 & 1.2737 & 1.2619 & 0.0094 & 1.2919 & 1.2723 & 0.0154 \\
\hline art & 1.2369 & 1.2362 & 0.0006 & 1.3377 & 1.2735 & 0.0504 & 1.3247 & 1.2801 & 0.0349 & 1.3273 & 1.2727 & 0.0429 \\
\hline equake & 1.2323 & 1.2309 & 0.0012 & 1.2200 & 1.1878 & 0.0271 & 1.2386 & 1.2139 & 0.0204 & 1.2349 & 1.2054 & 0.0245 \\
\hline facerec & 1.2338 & 1.2327 & 0.0009 & 1.3183 & 1.3058 & 0.0096 & 1.2941 & 1.2825 & 0.0090 & 1.2989 & 1.2856 & 0.0104 \\
\hline fma3d & 1.2559 & 1.2556 & 0.0003 & 1.2277 & 1.2126 & 0.0124 & 1.2563 & 1.2450 & 0.0091 & 1.2506 & 1.2339 & 0.0135 \\
\hline galgel & 1.2496 & 1.2491 & 0.0004 & 1.2612 & 1.2457 & 0.0125 & 1.2271 & 1.2152 & 0.0098 & 1.2339 & 1.2084 & 0.0211 \\
\hline lucas & 1.2598 & 1.2473 & 0.0100 & 1.3541 & 1.2130 & 0.1163 & 1.3371 & 1.2270 & 0.0897 & 1.3405 & 1.2213 & 0.0976 \\
\hline mesa & 1.3318 & 1.3311 & 0.0006 & 1.3668 & 1.2613 & 0.0836 & 1.3373 & 1.2576 & 0.0634 & 1.3432 & 1.2450 & 0.0789 \\
\hline mgrid & 1.2272 & 1.2267 & 0.0004 & 1.4826 & 1.2622 & 0.1746 & 1.4035 & 1.2393 & 0.1325 & 1.4193 & 1.2477 & 0.1375 \\
\hline sixtrack & 1.4039 & 1.3970 & 0.0049 & 1.4750 & 1.3877 & 0.0629 & 1.4617 & 1.3930 & 0.0493 & 1.4644 & 1.3927 & 0.0514 \\
\hline swim & 1.3084 & 1.3025 & 0.0045 & 1.5102 & 1.3524 & 0.1167 & 1.4813 & 1.3508 & 0.0966 & 1.4871 & 1.3585 & 0.0947 \\
\hline wupwise & 1.1406 & 1.1396 & 0.0009 & 1.2814 & 1.2626 & 0.0149 & 1.2200 & 1.2075 & 0.0104 & 1.2323 & 1.2067 & 0.0212 \\
\hline
\end{tabular}

Table 11: Upper bounds $c_{A}$ on the performance ratios achieved by $A \in\{M T F, T S, B I T, C O M B\}$ on the memory access traces, as implied by Theorems $1-4$. The values are compared to the experimentally observed competitiveness $c_{A}^{*}$, which is the actual cost incurred by $A$ to that of the pairwise optimum. The comparison yields a relative error $f_{c_{A}}$. 


\begin{tabular}{|c|c|c|c|c|c|}
\hline file & $\lambda$ & $c_{M T F}^{\lambda}$ & $f_{c_{M T F}^{\lambda}}$ & $c_{B I T}^{\lambda}$ & $f_{c_{B I T}^{\lambda}}$ \\
\hline bzip2 g7 & 0.2864 & 1.5547 & 0.2434 & 1.7500 & 0.3908 \\
\hline bzip2 g9 & 0.3076 & 1.5296 & 0.2243 & 1.7500 & 0.3919 \\
\hline bzip2 p7 & 0.2992 & 1.5394 & 0.2367 & 1.7500 & 0.3991 \\
\hline bzip2 p9 & 0.3193 & 1.5160 & 0.2189 & 1.7500 & 0.3955 \\
\hline bzip2 s7 & 0.2940 & 1.5456 & 0.2396 & 1.7500 & 0.3977 \\
\hline bzip2 s9 & 0.3151 & 1.5208 & 0.2208 & 1.7500 & 0.3891 \\
\hline crafty & 0.5245 & 1.3119 & 0.0974 & 1.6560 & 0.2408 \\
\hline eon cook & 0.3766 & 1.4529 & 0.2155 & 1.7264 & 0.4212 \\
\hline eon kajiya & 0.3800 & 1.4493 & 0.2199 & 1.7247 & 0.4326 \\
\hline eon rush & 0.3788 & 1.4506 & 0.2195 & 1.7253 & 0.4335 \\
\hline gap & 0.3843 & 1.4448 & 0.1600 & 1.7224 & 0.3178 \\
\hline gcc 166 & 0.4277 & 1.4008 & 0.1267 & 1.7004 & 0.2760 \\
\hline $\operatorname{gcc} 200$ & 0.4595 & 1.3703 & 0.1197 & 1.6852 & 0.2644 \\
\hline gcc expr & 0.4427 & 1.3863 & 0.1221 & 1.6932 & 0.2694 \\
\hline gcc integ & 0.4407 & 1.3882 & 0.1222 & 1.6941 & 0.2700 \\
\hline gcc scilab & 0.4555 & 1.3741 & 0.1203 & 1.6871 & 0.2676 \\
\hline gzip graphic & 0.3336 & 1.4997 & 0.1975 & 1.7498 & 0.3652 \\
\hline gzip log & 0.2363 & 1.6177 & 0.3233 & 1.7500 & 0.4404 \\
\hline gzip program & 0.3237 & 1.5109 & 0.2091 & 1.7500 & 0.3669 \\
\hline gzip random & 0.3367 & 1.4963 & 0.1857 & 1.7481 & 0.3463 \\
\hline gzip source & 0.3034 & 1.5344 & 0.2309 & 1.7500 & 0.3832 \\
\hline $\mathrm{mcf}$ & 0.5794 & 1.2663 & 0.0399 & 1.6331 & 0.1447 \\
\hline parser & 0.3841 & 1.4449 & 0.1747 & 1.7225 & 0.3498 \\
\hline perl diffmail & 0.4869 & 1.3450 & 0.1091 & 1.6725 & 0.2595 \\
\hline perl makerand & 0.5459 & 1.2938 & 0.0720 & 1.6469 & 0.2714 \\
\hline perl perfect & 0.5157 & 1.3195 & 0.0980 & 1.6598 & 0.2386 \\
\hline perl splitmail & 0.4903 & 1.3420 & 0.1053 & 1.6710 & 0.2498 \\
\hline twolf & 0.4697 & 1.3608 & 0.1223 & 1.6804 & 0.2713 \\
\hline vortex one & 0.4994 & 1.3339 & 0.0976 & 1.6669 & 0.2515 \\
\hline vortex two & 0.5031 & 1.3306 & 0.0954 & 1.6653 & 0.2478 \\
\hline vortex three & 0.5060 & 1.3280 & 0.0937 & 1.6640 & 0.2474 \\
\hline vpr place & 0.3319 & 1.5016 & 0.1718 & 1.7500 & 0.3225 \\
\hline vpr route & 0.3455 & 1.4865 & 0.1983 & 1.7432 & 0.3701 \\
\hline ammp & 0.3858 & 1.4432 & 0.1790 & 1.7216 & 0.3491 \\
\hline applu & 0.5229 & 1.3133 & 0.1510 & 1.6567 & 0.3571 \\
\hline apsi & 0.4896 & 1.3426 & 0.1500 & 1.6713 & 0.3245 \\
\hline art & 0.3621 & 1.4683 & 0.1878 & 1.7341 & 0.3547 \\
\hline equake & 0.2626 & 1.5840 & 0.2869 & 1.7500 & 0.4417 \\
\hline facerec & 0.3765 & 1.4530 & 0.1787 & 1.7265 & 0.3462 \\
\hline fma3d & 0.2436 & 1.6082 & 0.2809 & 1.7500 & 0.4057 \\
\hline galgel & 0.2266 & 1.6306 & 0.3054 & 1.7500 & 0.4401 \\
\hline lucas & 0.2641 & 1.5821 & 0.2684 & 1.7500 & 0.4263 \\
\hline mesa & 0.1889 & 1.6823 & 0.2639 & 1.7500 & 0.3916 \\
\hline mgrid & 0.3909 & 1.4379 & 0.1721 & 1.7189 & 0.3870 \\
\hline sixtrack & 0.2720 & 1.5723 & 0.1254 & 1.7500 & 0.2563 \\
\hline swim & 0.3872 & 1.4417 & 0.1069 & 1.7209 & 0.2740 \\
\hline wupwise & 0.4640 & 1.3661 & 0.1988 & 1.6830 & 0.3938 \\
\hline
\end{tabular}

Table 12: Competitive ratios $c_{M T F}^{\lambda}$ and $c_{B I T}^{\lambda}$ for the memory access traces, according to Corollaries 1 and 2. The values are compared to $c_{M T F}^{*}$ and $c_{B I T}^{*}$, respectively, yielding relative errors $f_{c_{M T F}^{\lambda}}$ and $f_{c_{B I T}^{\lambda}}$. 